\title{
Efficient computation of minimum-area rectilinear convex hull under rotation and generalizations*
}

\author{
Carlos Alegría-Galicia ${ }^{\dagger} \quad$ David Orden ${ }^{\ddagger} \quad$ Carlos Seara $^{\S} \quad$ Jorge Urrutia
}

口

\begin{abstract}
Let $P$ be a set of $n$ points in the plane. We compute the value of $\theta \in[0,2 \pi)$ for which the rectilinear convex hull of $P$, denoted by $\mathcal{R H}_{\theta}(P)$, has minimum (or maximum) area in optimal $O(n \log n)$ time and $O(n)$ space, improving the previous $O\left(n^{2}\right)$ bound. Let $\mathcal{O}$ be a set of $k$ lines through the origin sorted by slope and let $\alpha_{i}$ be the aperture angles of the $2 k$ sectors defined by every pair of two consecutive lines. Let $\Theta_{i}=\pi-\alpha_{i}$ and $\Theta=\min \left\{\Theta_{i}: i=1, \ldots, 2 k\right\}$. We further obtain: (1) Given a set $\mathcal{O}$ such that $\Theta \geq \frac{\pi}{2}$, we provide an algorithm to compute the $\mathcal{O}$-convex hull of $P$ in optimal $O(n \log n)$ time and $O(n)$ space, while if $\Theta<\frac{\pi}{2}$ the complexities are $O\left(\frac{n}{\Theta} \log n\right)$ time and $O\left(\frac{n}{\Theta}\right)$ space. (2) Given a set $\mathcal{O}$ such that $\Theta \geq \frac{\pi}{2}$, we compute and maintain the boundary of the $\mathcal{O}_{\theta}$-convex hull of $P$ for $\theta \in[0,2 \pi)$ in $O(k n \log n)$ time and $O(k n)$ space, or in $O\left(k \frac{n}{\Theta} \log n\right)$ time and $O\left(k \frac{n}{\Theta}\right)$ space if $\Theta<\frac{\pi}{2}$. (3) Finally, given a set $\mathcal{O}$ such that $\Theta \geq \frac{\pi}{2}$, we compute the $\mathcal{O}_{\theta}$-convex hull of $P$ of minimum (or maximum) area over all $\theta \in[0,2 \pi)$ in $O(k n \log n)$ time and $O(k n)$ space.
\end{abstract}

Keywords: Rectilinear convex hull, Restricted orientation convex hull, Minimum area.

\section{Introduction}

Restricted-orientation convexity is a generalization of traditional convexity that stems from the notion of restricted-orientation geometry, where the geometric objects under study comply with restrictions related to a fixed set of orientations. Restricted-orientation geometry started with the work of Güting [16] in the early eighties, as a generalization of the study of orthogonal polygons, whose edges are parallel to the coordinate axes.

Ortho-convexity or orthogonal convexity [20, 21, 25] was defined by defining as convex sets those whose intersection with any line parallel to a coordinate axis is either empty or connected.

\footnotetext{
*Extended abstracts related to this work appeared in the XIV Spanish Meeting on Computational Geometry [2] and in the $30^{\text {th }}$ European Workshop on Computational Geometry (EuroCG) 4].

${ }^{\dagger}$ Posgrado en Ciencia e Ingeniería de la Computación, Universidad Nacional Autónoma de México, calegria@uxmcc2.iimas. unam.mx.

${ }^{\ddagger}$ Departamento de Física y Matemáticas, Universidad de Alcalá, Spain, david.orden@uah.es. Research supported by project MTM2017-83750-P of the Spanish Ministry of Science (AEI/FEDER, UE).

${ }^{\S}$ Departament de Matemàtiques, Universitat Politècnica de Catalunya, Spain, carlos.seara@upc.edu. Research supported by projects Gen. Cat. DGR 2017SGR1640 and MINECO MTM2015-63791-R.

`Instituto de Matemáticas, Universidad Nacional Autónoma de México, urrutia@matem.unam.mx. Research supported in part by SEP-CONACYT 80268, PAPPIIT IN102117 Programa de Apoyo a la Investigación e Innovación Tecnológica UNAM.
}

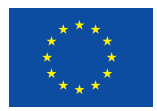

This project has received funding from the European Union's Horizon 2020 research and innovation programme under the Marie Skłodowska-Curie grant agreement No 734922. 
The corresponding convex hull of a set $P$ of $n$ points in the plane, called rectilinear convex hull of $P$ and denoted $\mathcal{R H}(P)$, has been extensively studied since its formalization in the early eighties and, together with the rectilinear convex hull of other geometric objects, it has been applied in several research fields, including illumination [1, polyhedron reconstruction [10, geometric search [27], and VLSI circuit layout design [28].

Researchers have also studied relations between rectilinear convex hulls of colored point sets [3], and developed generalizations of orthogonal convexity [14, 15, 19] along with related computational results [4, 5, 6]. Among these generalizations, it is particularly relevant the one by Fink and Wood [14, who defined $\mathcal{O}$-convexity calling convex sets to those whose intersection with any line parallel to one in a given set $\mathcal{O}$ of lines through the origin is either empty or connected. Thus, one can consider the $\mathcal{O}$-convex hull of a point set $P$, sometimes called $\mathcal{O}$-hull for short and denoted $\mathcal{O H}(P)$.

\subsection{Our work}

Bae et al. 8] considered the rectilinear convex hull of a set of point $P$ where convexity is defined by lines parallel to a rotation of the coordinate axis by an angle $\theta$, denoted by $\mathcal{R} \mathcal{H}_{\theta}(P)$ and called $\theta$-rectilinear convex hull. In fact, the coordinates will remain to be considered with respect to the horizontal and the vertical axes, although convexity will be considered with respect to the set $\mathcal{O}$ obtaining by rotating these axes by an angle $\theta \in[0,2 \pi)$. This is why these rotations will be mentioned as "axes" instead of "coordinate axes".

In $O\left(n^{2}\right)$ time and $O(n)$ space, Bae et al. computed the value of $\theta \in[0,2 \pi)$ such that the area of $\mathcal{R H}_{\theta}(P)$ is minimum (notice that this area is orientation-dependent, more details in Section 2 . However, the authors did not achieve to efficiently maintain the linear number of events where the structure of $\mathcal{R H}_{\theta}(P)$ changes as the value of $\theta$ is increased from 0 to $2 \pi$.

In the present paper, we improve the results of Bae et al. [8] by providing an optimal $O(n \log n)$ time and $O(n)$ space algorithm. Our scheme for such an improvement is to maintain two structures: The set of vertices of $\mathcal{R H}_{\theta}(P)$, which has to be updated at every change arising during the rotation $\theta \in[0,2 \pi)$ and the set of overlapping intervals, defined as the angular intervals contained in $[0,2 \pi)$ where opposite staircases of the boundary of $\mathcal{R H}_{\theta}(P)$ overlap with each other (more details in Section 2.2.1). Dealing with the set of vertices was achieved, indirectly, by Díaz-Báñez et al. [12] in optimal $O(n \log n)$ time and $O(n)$ space, while designing an algorithm to fit an orthogonal chain to a point set in the plane. Dealing with the set of overlapping intervals in $O(n \log n)$ time and $O(n)$ space is one of the main contributions in the current paper. In Section 2, we show how to maintain these two structures and design the optimal $O(n \log n)$ time and $O(n)$ space algorithm for this improvement.

In Section 3 we focus on $\mathcal{O}$-convex sets, i.e., sets for which the intersection with any line parallel to a line in $\mathcal{O}$ is either empty or connected. We design algorithms to compute the $\mathcal{O}_{-}$ convex hull of a set $P$ of $n$ points, denoted by $\mathcal{O H}(P)$; and also we generalize to $\mathcal{O}$-convexity the rotation in Bae et al. [8] considering the $\mathcal{O}_{\theta}$-convexity, i.e., the convexity defined by the set $\mathcal{O}$ of $k$ lines rotated by an angle $\theta$, leading to $\mathcal{O H}_{\theta}(P)$. One of the problems will be computing and maintaining the boundary of $\mathcal{O H}_{\theta}(P)$ during a complete rotation for $\theta \in[0,2 \pi)$. Some of the algorithms are sensitive to the following two parameters. The first parameter will be the cardinal $k \geq 2$ of a set $\mathcal{O}=\left\{\ell_{1}, \ldots, \ell_{k}\right\}$ of lines through the origin sorted by slope. The $O(k \log k)$ time complexity for sorting the lines in $\mathcal{O}$ can be added to the complexity of the upcoming algorithms; nevertheless, as we will see below, the number $k$ will be smaller than the number $n$ of input points of the problems, and thus, $O(k \log k)$ will be in $O(n \log n)$. However, the complexity of some of our algorithms can be sensitive to $k$, which will appear as a multiplicative factor. For the second parameter, let $\alpha_{i}$ be the aperture angles of the $2 k$ sectors defined by two consecutive 
lines in $\mathcal{O}$ and let $\Theta_{i}=\pi-\alpha_{i}$. The second parameter will be $\Theta=\min \left\{\Theta_{i}: i=1, \ldots, 2 k\right\}$, which measures how "well distributed" are the the orientations of the $k$ lines of $\mathcal{O}$ into the unit circle and, in particular, we will distinguish whether $\Theta \geq \frac{\pi}{2}$ or $\Theta<\frac{\pi}{2}$.

\subsection{Contributions and relevance}

The main contributions of this work are the following:

- We improve the $O\left(n^{2}\right)$ time complexity from Bae et al. [8, computing in optimal $O(n \log n)$ time and $O(n)$ space the value of $\theta \in[0,2 \pi)$ for which $\mathcal{R H}_{\theta}(P)$ has minimum (or maximum) area, also returning $\mathcal{R H}_{\theta}(P)$ (Theorem 5).

- Given a set $\mathcal{O}$ of $k$ lines such that $\Theta \geq \frac{\pi}{2}$, we provide an algorithm to compute $\mathcal{O H}(P)$, in optimal $O(n \log n)$ time and $O(n)$ space (Theorem 6).

- Given a set $\mathcal{O}$ of $k$ lines such that $\Theta<\frac{\pi}{2}$, we provide an algorithm to compute $\mathcal{O H}(P)$ in $O\left(\frac{n}{\Theta} \log n\right)$ time and $O\left(\frac{n}{\Theta}\right)$ space (Theorem 7$)$.

- We generalize to $\mathcal{O}$-convexity the rotation in Bae et al. 8] showing that, for a set $\mathcal{O}$ of $k$ lines, computing and maintaining the boundary of $\mathcal{O H}_{\theta}(P)$ during a complete rotation for $\theta \in[0,2 \pi)$ can be done in $O(k n \log n)$ time and $O(k n)$ space (Theorem 8 for $\Theta \geq \frac{\pi}{2}$ or in $O\left(k \frac{n}{\Theta} \log n\right)$ time and $O\left(k \frac{n}{\Theta}\right)$ space (Theorem 9 for $\Theta<\frac{\pi}{2}$.

- As a consequence, for the two cases $\Theta \geq \frac{\pi}{2}$ or $\Theta<\frac{\pi}{2}$, computing an interval of $\theta$ such that the boundary of $\mathcal{O H}_{\theta}(P)$ has minimum number of staircases, or minimum number of steps, or it is connected, or it has the minimum number of connected components, can be done in $O(k n \log n)$ time and $O(k n)$ space, or in $O\left(k \frac{n}{\Theta} \log n\right)$ time and $O\left(k \frac{n}{\Theta}\right)$ space, respectively (Corollaries 1 and 2 ).

- Given a set $\mathcal{O}$ composed by two non-perpendicular lines through the origin, we show that computing and maintaining the boundary of $\mathcal{O H}_{\theta}(P)$ during a complete rotation for $\theta \in[0,2 \pi)$ can be done in $O\left(\frac{n}{\Theta} \log n\right)$ time and $O\left(\frac{n}{\Theta}\right)$ space, where $\Theta$ is the smallest aperture angle of the sectors defined by the two lines (Corollary 3 ).

- Given a set $\mathcal{O}$ of $k$ lines such that $\Theta \geq \frac{\pi}{2}$, we provide an algorithm to compute $\mathcal{O H}_{\theta}(P)$ with minimum (or maximum) area over all $\theta \in[0,2 \pi)$ in $O(k n \log n)$ time and $O(k n)$ space (Theorem 12 ).

For possible applications, it is relevant to note that rotation-dependent and minimum area enclosing shapes are commonly used in form-shape analysis [11, 13, 24, 29, as well as in feature classification [18, 26].

\section{Rectilinear hull of a point set}

Let $P=\left\{p_{1}, \ldots, p_{n}\right\}$ be a set of $n$ points in the plane, in general position. Let $\mathcal{C H}(P)$ denote the convex hull of $P$ and let $V=\left\{p_{1}, \ldots, p_{h}\right\}$ be the set of vertices of its boundary $\partial(\mathcal{C H}(P))$, as we meet them in counterclockwise order (starting at an arbitrary vertex $p_{1}$ ). Further, let $E=\left\{e_{1}, \ldots, e_{h}\right\}$ be the set of edges of $\partial(\mathcal{C H}(P))$, where $e_{i}=\overline{p_{i} p_{i+1}}$ and the indices are taken modulo $h$. 
An open quadrant in the plane is the intersection of two open half planes whose supporting lines are parallel to the $x$ - and $y$ - axes. Such an open quadrant is said to be $P$-free if it contains no element of $P$. The rectilinear convex hull of $P[22$ is the set:

$$
\mathcal{R H}(P)=\mathbb{R}^{2} \backslash \bigcup_{W \in \mathcal{W}} W
$$

where $\mathcal{W}$ denotes the set of all $P$-free open quadrants. See Figure 1 , left, for an example. It is interesting to note that, whith this definition, the rectilinear convex hull might be disconnected [22].
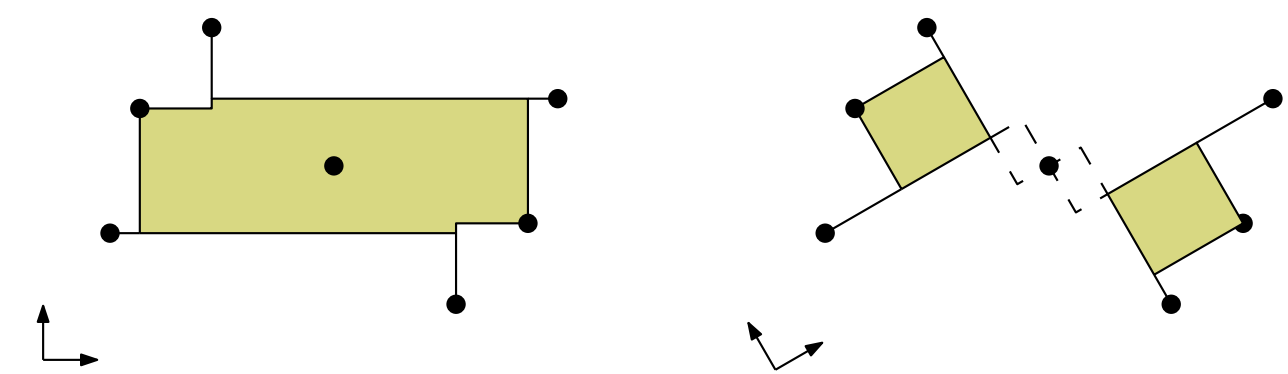

Figure 1: Left: The rectilinear convex hull $\mathcal{R H}(P)$ of a point set $P$. Right: The rectilinear convex hull $\mathcal{R H}_{\theta}(P)$ of the same point set for $\theta=\pi / 6$.

As in Bae et al. 8], we will also consider the rectilinear convex hull when rotating the axes by an angle $\theta$, also called the $\theta$-rectilinear convex hull of $P$ :

$$
\mathcal{R H}_{\theta}(P)=\mathbb{R}^{2} \backslash \bigcup_{W_{\theta} \in \mathcal{W}_{\theta}} W_{\theta},
$$

where $\mathcal{W}_{\theta}$ denotes the set of all $P$-free open $\theta$-quadrants, i.e., the open quadrants obtained when the axes are rotated by $\theta$. Figure 1, right, shows an example, where the $\pi / 6$-rectilinear convex hull happens to be disconnected.

\subsection{Computing and maintaining $\mathcal{R H}_{\theta}(P)$}

In this subsection we describe an algorithm to compute and maintain $\mathcal{R H}_{\theta}(P)$, together with all its relevant features, over all the rotations of the axes by an angle $\theta \in[0,2 \pi)$. The algorithm works in optimal $O(n \log n)$ time and $O(n)$ space, thus improving the $O\left(n^{2}\right)$ time complexity achieved by Bae et al. [8].

Let us start with the fixed value $\theta=0$. Given two points $p_{i}, p_{j} \in P$, the dominance relation $p_{i}$ dominates $p_{j}$, denoted by $p_{j} \prec p_{i}$, is defined to be fulfilled when $x_{j} \leq x_{i}$ and $y_{j} \leq y_{i}$. This relation is a partial order in $P$, and a point $p_{i} \in P$ is called maximal if there does not exist $p_{j} \in P$ such that $i \neq j$ and $p_{i} \prec p_{j}$. The Set Maxima Problem [23] consists of finding all the maximal points of $P$ under this dominance $\prec$. Note that the condition above is equivalent to $p_{j}$ being contained in the open quadrant with apex $p_{i}$ which is a translation of the third quadrant. Analogous set maxima problems can be defined considering the remaining three quadrants, and the maxima problem for $P$ with respect to any of the four quadrants can be solved optimally in $O(n \log n)$ time and $O(n)$ space [23], where each set of maximal points has a total ordering and can be organized as a height balanced search tree. These sets of maxima points for $P$ 
with respect to the four quadrants provide the points of $P$ on the boundary $\partial(\mathcal{R H}(P))$ of the rectilinear convex hull of $P$ for $\theta=0$. See again Figure 1, left.

Let us move to the case where the axes are rotated as in Bae et al. [8]. The set $\mathcal{V}_{\theta}(P)$ of points of $P$ lying on the boundary $\partial\left(\mathcal{R H}_{\theta}(P)\right)$ of the rotated $\mathcal{R} \mathcal{H}_{\theta}(P)$ will be called the set of vertices of $\mathcal{R H}_{\theta}(P)$. As above, for any fixed $\theta$ the computation of $\mathcal{R} \mathcal{H}_{\theta}(P)$ reduces to solving four set maxima problems, since

$$
\mathcal{V}_{\theta}(P)=V_{\theta}(P) \cup V_{\theta+\frac{\pi}{2}}(P) \cup V_{\theta+\pi}(P) \cup V_{\theta+\frac{3 \pi}{4}}(P),
$$

for $V_{\theta}$ being the set of maximal points of $P$ with respect to the $\theta$-quadrant defined rotating by $\theta$ the $x$ - and $y$ - axes $\left[8,12,22\right.$. In order to keep track of the changes in the set $\mathcal{V}_{\theta}(P)$ of vertices of $\mathcal{R H}_{\theta}(P)$ while changing $\theta$ in $[0,2 \pi)$, we can use results from Avis et al. [7] and Díaz-Báñez et al. [12] as follows.

Every point $p \in \mathcal{V}_{\theta}(P)$ is the apex of a $P$-free open $\theta$-quadrant in $\mathcal{W}_{\theta}$. Figure 2(a) shows a point $p$ that is in $\mathcal{V}_{\theta}(P)$ (i.e., a vertex of $\mathcal{R H}_{\theta}(P)$ ) for all $\theta$ in the interval $I_{p}=\left[\theta^{\prime}, \theta^{\prime \prime}\right)$. The endpoints of $I_{p}$ mark the in and out events of $p$, i.e., the values of $\theta$ when $p$ starts and stops being in $\mathcal{V}_{\theta}(P)$. Because of the general position assumption for $P$, a point $p \in P$ can have at most three intervals $I_{p}$ for which it is a vertex of $\mathcal{R H}_{\theta}(P)$, i.e., for which $p \in \mathcal{V}_{\theta}(P)$.

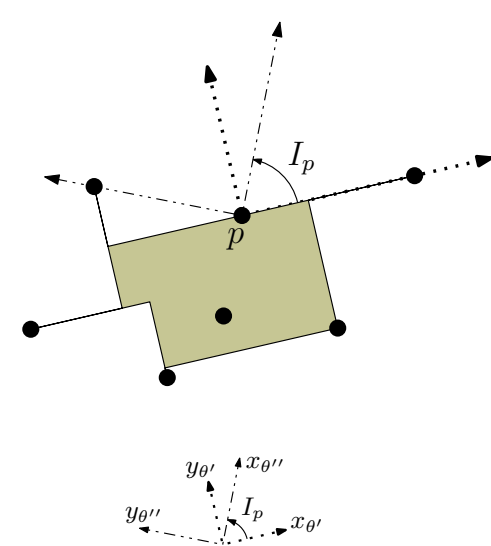

(a)
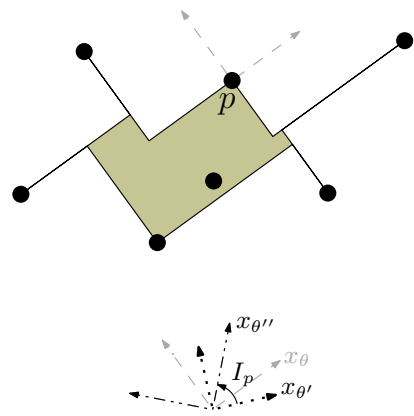

(b)
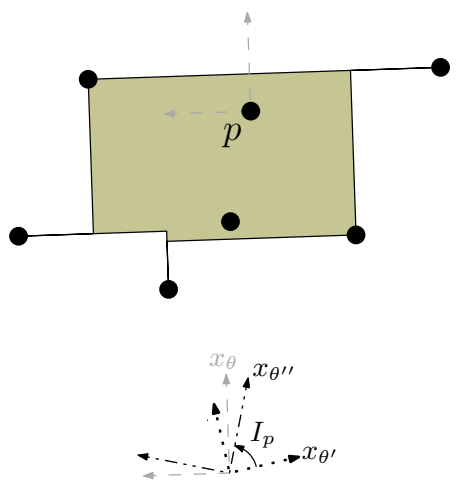

(c)

Figure 2: (a) $I_{p}=\left[\theta^{\prime}, \theta^{\prime \prime}\right)$, together with $P$-free open $\theta^{\prime}$ - and $\theta^{\prime \prime}$-quadrants (rotations of the first quadrant) with apex $p$. (b) Situation for a $\theta \in I_{p}$ and $P$-free open $\theta$-quadrant with apex $p$. (c) Situation for a $\theta \notin I_{p}$ and non- $P$-free open $\theta$-quadrant with apex $p$.

The following Theorem 1 is not stated explicitly as a result in [12], but it appears as a main step of an algorithm for a fitting problem. The proof is obtained by, first, computing the set of intervals $I_{p}$ as above for which the points $p \in P$ are maximal with respect to some $\theta$-quadrant (using a result from Avis et al. [7]), then, computing the ordered set of in- and out-events while $\theta$ increases from 0 to $2 \pi$ (performing a line sweep with four lines to obtain the maximal points of $P$ for each of the four $\theta$-quadrants). The reader is referred to [12] for further details.

Theorem 1 (Díaz-Bañez et al. [12]). Computing and maintaining the $\theta$-rectilinear convex hull $\mathcal{R H}_{\theta}(P)$ while $\theta$ increases from 0 to $2 \pi$ can be done in optimal $O(n \log n)$ time and $O(n)$ space. 


\subsection{Finding the value of $\theta$ for which $\mathcal{R H}_{\theta}(P)$ has minimum area}

For a fixed value of $\theta$, we can compute the area of $\mathcal{R} \mathcal{H}_{\theta}(P)$ using the fact that

$$
\operatorname{area}\left(\mathcal{R H}_{\theta}(P)\right)=\operatorname{area}(\mathcal{P}(\theta))-\operatorname{area}\left(\mathcal{P}(\theta) \backslash \mathcal{R H}_{\theta}(P)\right),
$$

where $\mathcal{P}(\theta)$ denotes the filled polygon having the points in $\mathcal{V}_{\theta}(P)$ as vertices and an edge connecting two vertices if they are consecutive elements in $\mathcal{V}_{\theta}(P)$, see Figure 3 . We will compute the area of $\mathcal{P}(\theta) \backslash \mathcal{R H}_{\theta}(P)$ by decomposing it into two types of regions: (i) The triangles defined by every pair of consecutive elements in $\mathcal{V}_{\theta}(P)$, and (ii) the rectangular overlaps between two triangles that make $\mathcal{R H}_{\theta}(P)$ being disconnected, see again Figure 3.

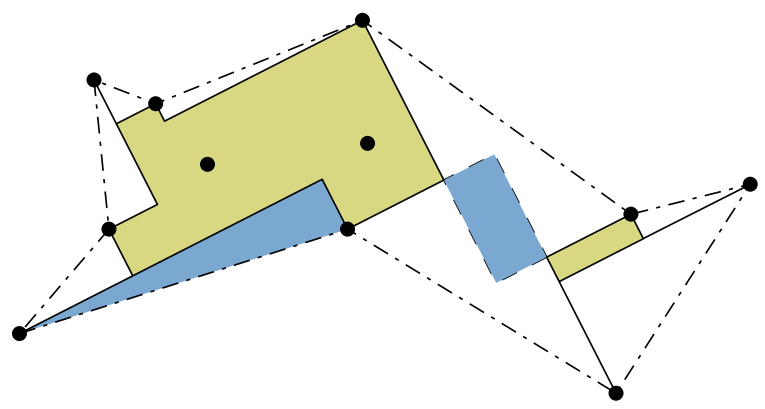

Figure 3: Computing the area of $\mathcal{R H}_{\theta}(P)$. The polygon $\mathcal{P}(\theta)$ is bounded by the dash-dotted line. A triangle and a rectangular overlap are filled with blue.

By Theorem 1, the triangles in (i) above can be maintained in optimal $O(n \log n)$ time and $O(n)$ space. While $\theta$ increases from 0 to $2 \pi$, the set $\mathcal{V}_{\theta}(P)$ of points on $\partial\left(\mathcal{R} \mathcal{H}_{\theta}(P)\right)$ changes at the values of $\theta$ where a point of $P$ becomes (resp. is no longer) a vertex of $\mathcal{R} \mathcal{H}_{\theta}(P)$. We call these angles in (resp. out) events.

The rest of this subsection will deal with the rectangles in (ii), showing how to maintain the set $\mathcal{S}_{\theta}(P)$ of rectangular overlaps that, analogously to $\mathcal{V}_{\theta}(P)$, changes at overlap (resp. release) events where such a rectangular overlap appears (resp. disappears). See Figure 4. It is important to notice that there exist point configurations for which overlap and release events do not coincide with vertex events [8] and, hence, the computations of $\mathcal{V}_{\theta}(P)$ and $\mathcal{S}_{\theta}(P)$ are independent.

\subsubsection{On overlap and release events}

Let us label the points of $P$ in $V_{\theta}(P)$ (recall Equation 1) as $v_{1}, \ldots, v_{m}$ in increasing order according to the rotation $x_{\theta}$ of the $x$-axis. Let $W_{\theta}^{i}$ denote the $P$-free $\theta$-quadrant supported by two points $v_{i}, v_{i+1} \in V_{\theta}(P)$, see Figure $4(\mathrm{a})$, and proceed analogously for $V_{\theta+\frac{\pi}{2}}(P), V_{\theta+\pi}(P)$, and $V_{\theta+\frac{3 \pi}{4}}(P)$. The $P$-free $\theta$-quadrants obtained, that define the boundary of $\mathcal{R H}_{\theta}(P)$, will be called extremal. We say that two such $\theta$-quadrants with a difference of $\pi$ in the subindex, e.g., $W_{\theta}^{i}$ and $W_{\theta+\pi}^{j}$, are opposite, see again Figure $4(\mathrm{a})$. When the intersection of two opposite extremal $\theta$-quadrants is nonempty, as in Figure 4(b) where $W_{\theta}^{i} \cap W_{\theta+\pi}^{j} \neq \emptyset$, we say that they overlap, and we denote their intersection by $S_{\theta}(i, j)$. Thus, the set $\mathcal{S}_{\theta}(P)$ of rectangular overlaps defined above is composed by these $S_{\theta}(i, j)$.

Recall, from the beginning of Subsection 2.1, that for a fixed value of $\theta$ the set $\mathcal{V}_{\theta}(P)$ of vertices can be computed in optimal $O(n \log n)$ time and $O(n)$ space. For that fixed $\theta$, the set 


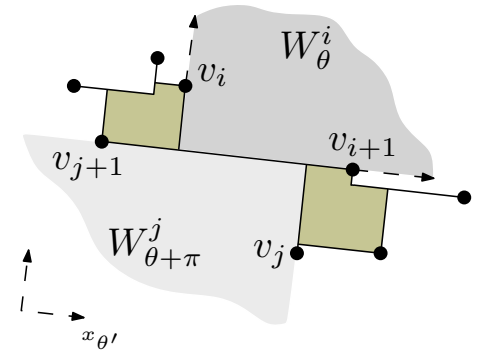

(a)

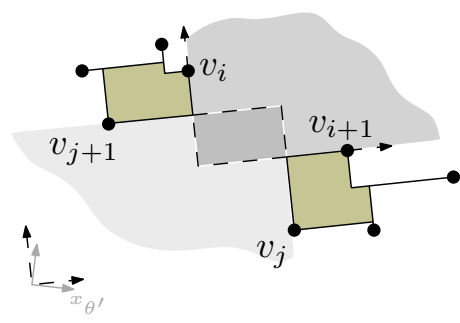

(b)

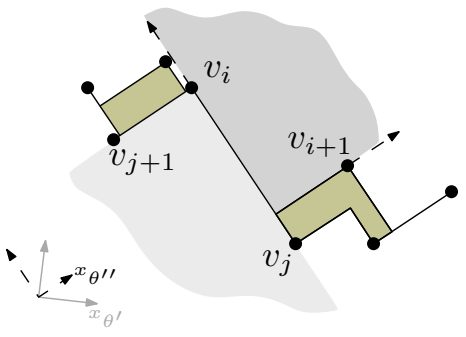

(c)

Figure 4: (a) Overlap event (an overlap begins at angle $\theta^{\prime}$ ). (b) Overlap, which disconnects $\mathcal{R H}_{\theta}(P)$. (c) Release event (an overlap ends at angle $\theta^{\prime \prime}$ ).

$\mathcal{S}_{\theta}(P)$ of rectangular overlaps can be computed from $\mathcal{V}_{\theta}(P)$ in $O(n)$ time. In the following, we show how to efficiently maintain the set $\mathcal{S}_{\theta}(P)$ while $\theta$ increases from 0 to $2 \pi$. We now justify why overlaps were defined above only for opposite extremal $\theta$-quadrants.

Lemma 1. If two extremal $\theta$-quadrants have nonempty intersection, then they have to be opposite. When this happens, $\mathcal{R H}_{\theta}(P)$ gets disconnected.

Proof. In any pair of non-opposite $\theta$-quadrants, one of them contains a ray parallel to a bounding ray of the other one. Since every extremal $\theta$-quadrant is supported by at least two points of $P$ (recall that it defines part of the boundary of $\mathcal{R H}_{\theta}(P)$ ), if a pair of non-opposite extremal $\theta$-quadrants had nonempty intersection, that would imply one of them not being $P$-free, a contradiction. See again Figure 4(b).

This property will be useful in the next two subsections, where we will show that the number of overlap and release events is linear and, then, we will illustrate an algorithm to compute them in an optimal way.

\subsubsection{The chain of arcs}

Let the chain of arcs of $P$, denoted by $\mathcal{A}(P)$, be the curve composed by the points $a$ in the plane which are apexes of a $P$-free extremal $\theta$-quadrant $W^{a}$ for some $\theta \in[0,2 \pi)$. Notice that $W^{a}$ is supported by at least two points of $P$. The sub-chain associated to an edge $e_{i}$ of $\partial(\mathcal{C H}(P))$ will be defined as the curve $A_{e_{i}}$ composed by those points $a$ such that $W^{a}$ intersects $e_{i}$. See Figure 5 , left. This sub-chain $A_{e_{i}}$ is monotone with respect to $e_{i}$, since it is composed by arcs of circles, which have to be monotone in order for $W^{a}$ to intersect $e_{i}$, and two consecutive monotone arcs whose extremal $\theta$-quadrants intersect $e_{i}$ can only form a monotone curve. Finally, since a sub-chain may have vertices not belonging to $P$, we call link to the part of a sub-chain which lies between two points of $P$. See Figure 5 , right.

Note that, if a pair of opposite extremal $\theta$-quadrants generates an overlapping region, then their apexes lie on intersecting links and the rectangular overlap lies in the area bounded by the intersection of the two links. See again Figure 5, left. Hence, in order to prove that the set $\mathcal{S}_{\theta}(P)$ of overlapping regions can be maintained in linear time and space, we will prove that the number of overlap and release events is linear by proving that there is a linear number of intersections between links. 

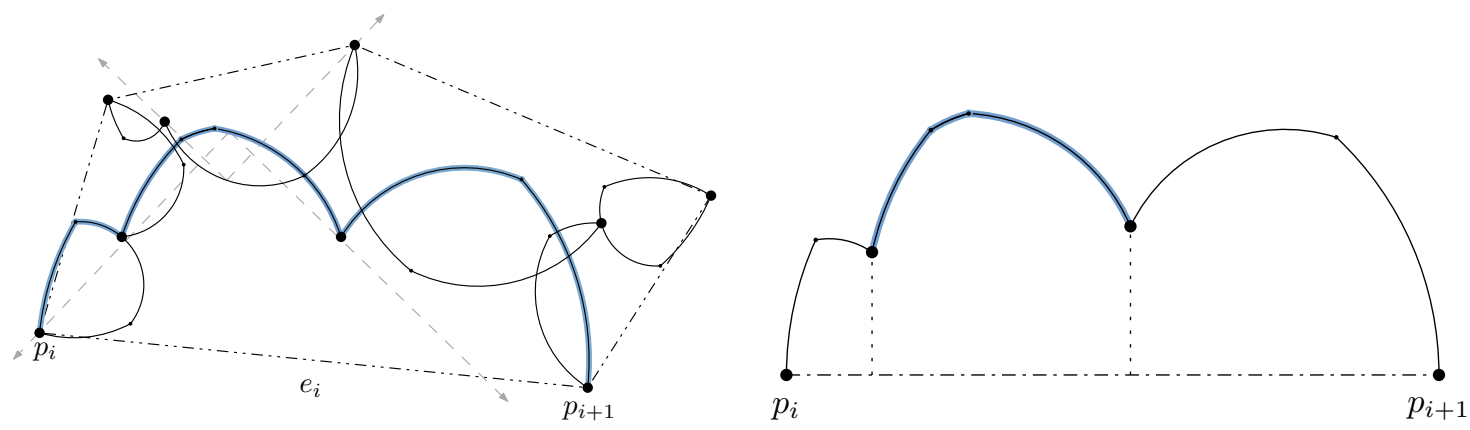

Figure 5: Left: The arc-chain of $P$, highlighting the sub-chain associated to $e_{i}$. Right: Highlighted, a link of that sub-chain.

\subsubsection{The number of intersections between links is in $O(n)$}

Let us outline the flow of ideas in this subsection. We will construct a weighted graph, whose vertices are the sub-chain disks, defined as disks that have as diameter an edge of $\partial(\mathcal{C H}(P))$. The edges of the weighted graph will join those sub-chain disks whose corresponding sub-chains intersect. The number of intersections will be, precisely, the weight of the edge. Then, the total number of intersections equals the sum of weights, which we are proving to be linear.

Each point $p \in P$ can be in at most four sub-chain disks, because $p$ can be the apex of at most four $P$-free wedges of size $\frac{\pi}{2}$ (actually, of at most three when considering general position). Thus, each point $p \in P$ can be in the intersection of at most $\left(\begin{array}{l}4 \\ 2\end{array}\right)=6$ pairs of sub-chain disks, therefore contributing to the weight of at most 6 edges of the weighted graph. We will prove that the weight of every edge in the graph is linear on the number of points from $P$ contained in the corresponding sub-chain disks (Theorem 2). Therefore, the sum of weights in the graph will be linear on the total number of points in $P$, as aimed.

We first need a series of three lemmas:

Lemma 2. For any three points a,b,c appearing from left to right on a link, the angle $\angle a b c$ lies in $\left[\frac{\pi}{2}, \pi\right)$. In particular, every link with endpoints $p, q \in P$ is contained in the disk of diameter $\overline{p q}$, which will be called its link disk.

Proof. Since $p, q$ are the endpoints of the link, hence consecutive points of $P$ along the chain, it turns out that $b \notin P, W^{b}$ being a $P$-free extremal $\theta$-quadrant. That $\angle a b c \geq \frac{\pi}{2}$ follows from $a, c$ not being in the interior of $W^{b}$ (otherwise this would not be $P$-free, either because some of $a, c$ is in $P$ or because one of the points of $P$ supporting the extremal $\theta$-quadrants with apexes $a, c$ is in the interior of $\left.W^{b}\right)$. That $\angle a b c<\pi$ follows from the orthogonal projections of $p$ and $q$ over the corresponding edge of $\partial(\mathcal{C H}(P))$ being inside the intersection of that edge with $W^{b}$. See Figure 6 .

In the following lemma we identify the diameter of a link with the diameter of its link disk.

Lemma 3. Consider the link disks in the two sub-chains associated to a pair of edges of the boundary $\partial(\mathcal{C H}(P))$. The link disk $D$ of the smallest diameter can be intersected by at most five links from the other sub-chain $A_{e}$.

Proof. Let $R$ be the strip bounded by the lines that orthogonally project $D$ over the edge $e$ associated to the sub-chain $A_{e}$. Because of the monotonicity, only the part of the sub-chain being inside $R$ can intersect $D$ (see Figure 7, left). 


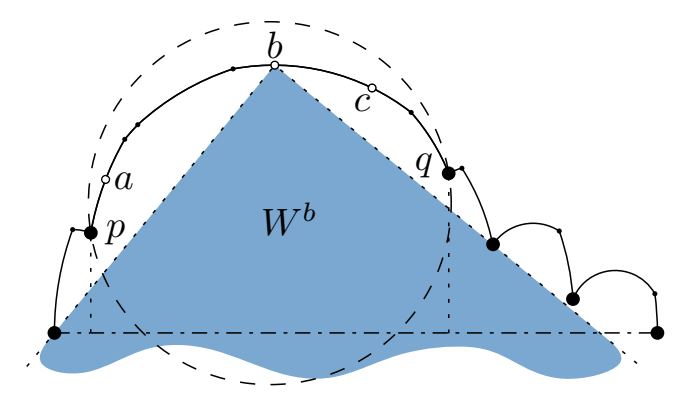

Figure 6: Illustration of Lemma 2 .
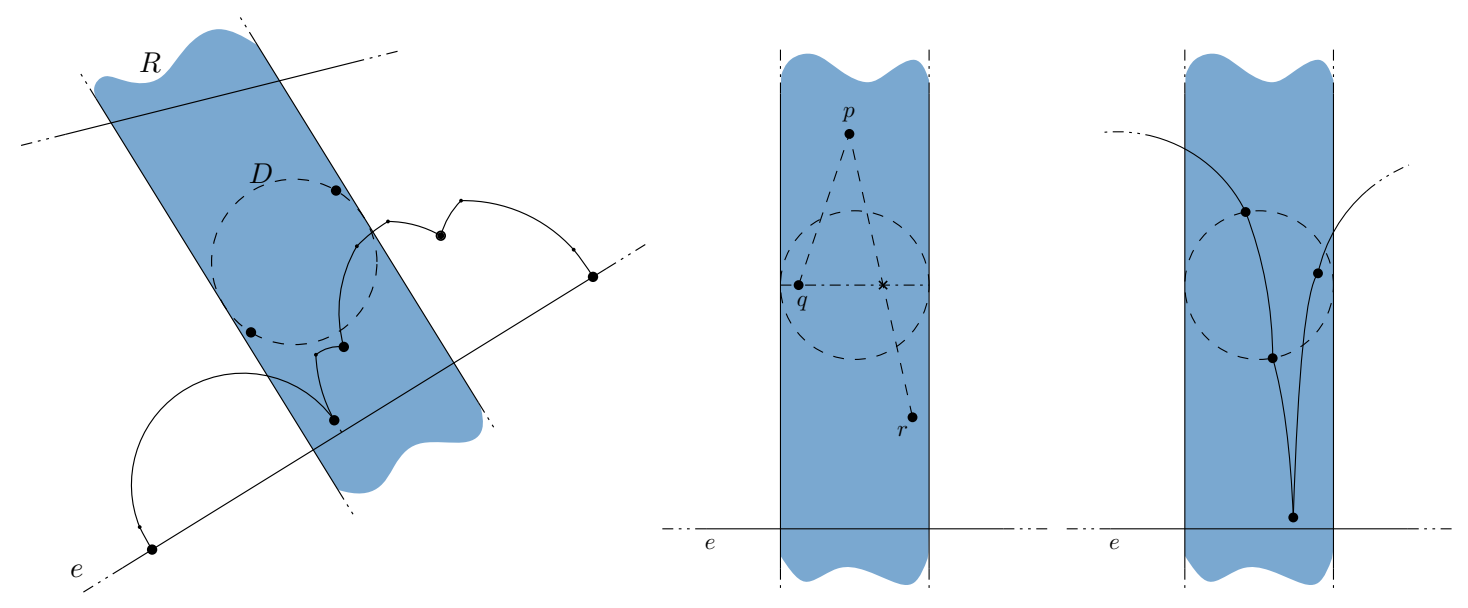

Figure 7: Left: Only the part of the sub-chain being inside $R$ can intersect $D$. Middle: There are no peaks at points of $P$ inside $R$. Right: At most 5 links intersect $D$.

If no arc in the sub-chain $A_{e}$ has endpoints inside $R$, then at most one link can intersect $D$. Otherwise, we will see that the sub-chain $A_{e}$ has no peaks at points of $P$ inside $R$ : If there were a peak $p \in R$, let $q, r$ be its neighbors, being $r$ the one closer to the edge $e$. The segment obtained by intersecting the parallel to $e$ through $q$ with the strip $R$, determines a disk which does not contain the peak $p$, since the length of $\overline{p q}$ equals the diameter of a link-disk and, hence, has to be greater than the diameter of $D$, which equals the width of $R$. See Figure 7, middle. Then $\angle q p r<\frac{\pi}{2}$, a contradiction with $W^{p}$ being $P$-free.

Since $A_{e}$ has no peaks at points of $P$ inside $R$, it can have at most one valley inside $R$ and, therefore, at most five links from $A_{e}$ can intersect $D$, since this is inside $R$. See Figure 7 , right.

Lemma 4. There are $O(n)$ pairs of intersecting links in the two sub-chains associated to a pair of edges of $\partial(\mathcal{C H}(P))$.

Proof. Let $\mathcal{L}$ be the list of all those links, ordered by increasing diameter. From Lemma 3 , the first link in $\mathcal{L}$ is intersected by at most five of the remaining links in $\mathcal{L}$. By removing this link from $\mathcal{L}$, we get that the next link in the list is also intersected by at most a constant number of links. As there is a linear number of arcs and each arc belongs to a single link, there is also a linear number of elements in $\mathcal{L}$. Therefore, by recursively removing the link with the smallest diameter from $\mathcal{L}$, the total number of intersecting pairs adds up to $O(n)$.

We are now ready to prove the main result of this section, Theorem 2, which implies that 
the weight of every edge in the weighted graph defined above is linear on the number of points from $P$ contained in the corresponding sub-chain disks.

Theorem 2. There are $O(n)$ intersection points between the links in the two sub-chains associated to a pair of edges of $\partial(\mathcal{C H}(P))$. Hence, the number of overlap and release events is in $O(n)$.

Proof. Because of the monotonicity, we know that two links within the same sub-chain can intersect only at one of their endpoints. By Lemma 4, we just have to prove that links from two different sub-chains intersect at most twice.

Suppose that there exist at least three intersection points $a, b, c$ between two links from sub-chains associated to $e_{i}$ and $e_{s}$. Without loss of generality, assume that $a, b, c$ appear from left to right on the link associated to $e_{i}$. Note that, then, they also appear from left to right on the link associated to $e_{s}$, since otherwise at least one of the points cannot belong to this link, as the three of them would form an angle either smaller than $\frac{\pi}{2}$ (Figure $8(\mathrm{a})$ or greater than $\pi$ (Figure 8(b) , in contradiction with Lemma 2 .

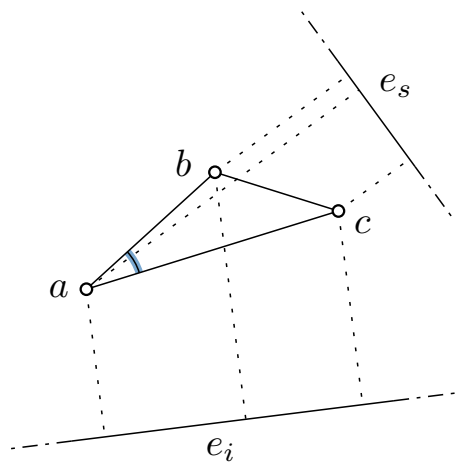

(a)

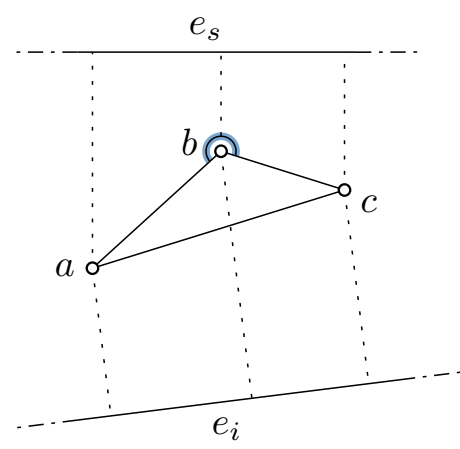

(b)

Figure 8: (a) $\angle c a b<\frac{\pi}{2}$, (b) $\angle c b a>\pi$.

Let $e_{l}$ and $e_{m}$ be respectively, the edges of $\partial(\mathcal{C H}(P))$ intersected by the rays from $b$ passing through $a$ and $c$. While traversing the edge set of $\partial(\mathcal{C H}(P))$ in counterclockwise direction, $e_{s}$ lies between either $e_{l}$ and $e_{i}$, or $e_{i}$ and $e_{m}$ (see Figure 9(a)p. Consider $e_{s}$ to be in the first case (the argument for the second case is symmetric) and denote with $\ell$ the line perpendicular to $e_{i}$ passing through $a$. Since $W^{a}$ is a maximal wedge bounded by rays intersecting $e_{i}$, as in the proof of Lemma 2, $W^{a}$ does not contain any other point from the link associated to $e_{i}$ (see Figure 9(b)]. Note that $c$ and $p_{s+1}$ are in opposite sides of $\ell$ and are not contained in $W^{a}$ and, thus, $\angle p_{s+1} a c \geq \frac{\pi}{2}$ and $\angle a c p_{s+1}<\frac{\pi}{2}$. Since $a, c, p_{s+1}$ appear from left to right on the link associated to $e_{s}$, we get from Lemma 2 that $c$ cannot belong to $A_{s}$.

\subsubsection{Computing the sequence of overlap and release events}

Next, we outline the algorithm to compute the sequence of overlap and release events.

\section{EVENT-SEQUENCE ALGORITHM}

1. Compute the chain of arcs of $P$.

Each arc should be described by the points supporting the corresponding extremal $\theta$ quadrant and the angular interval defined by these points, called the tracing interval. The elements in $\mathcal{A}(P)$ should be grouped by links. 


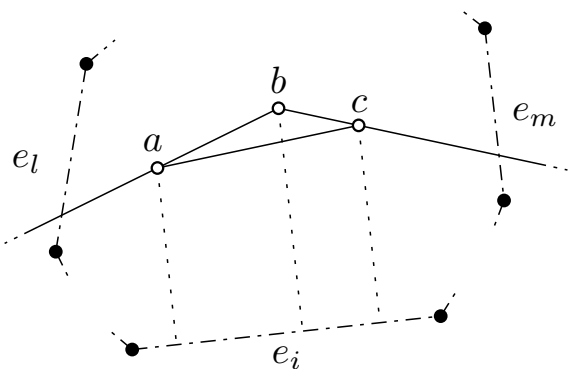

(a)

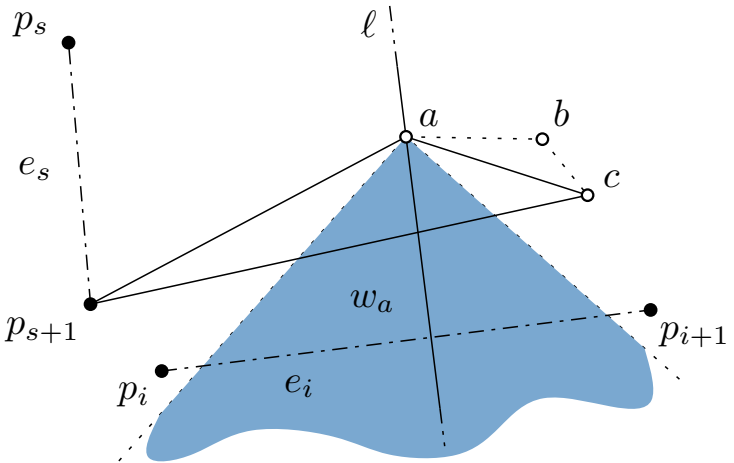

(b)

Figure 9: (a) Valid relative positions of the edge $e_{s},(\mathrm{~b}) c$ is not contained in $A_{s}(P)$ as $\angle a c p_{s+1}<\frac{\pi}{2}$.

(a) At each insertion event, at most two arcs are generated, and at most one arc is interrupted. Pointers should be set up from the interrupted arc to the ones just generated. If an extreme of a new arc is a point in $P$, a new link should be initialized with the respective arc.

(b) At each deletion event, at most one arc is generated, and at most two arcs are interrupted. One of the interrupted arcs will be always ending at a point in $P$, so a link is completed. As before, pointers from the interrupted to the newly created arcs should be set up.

2. Color arcs.

Traverse $\mathcal{A}(P)$ in such way that the vertices of $\partial(\mathcal{C H}(P))$ are visited in counterclockwise order, while assigning the following colors to each arc: red if its subchain corresponds to an edge in the upper chain $\partial(\mathcal{C H}(P))$, and blue otherwise (see Figure 10). Note that regardless of the value of $\theta$, a pair of extremal $\theta$-quadrants intersecting an edge in the upper chain (resp. lower chain) of $\partial(\mathcal{C H}(P))$ are not opposite to each other. Then, if there is an intersection between monochromatic links, such an intersection does not correspond to overlapping extremal $\theta$-quadrants.

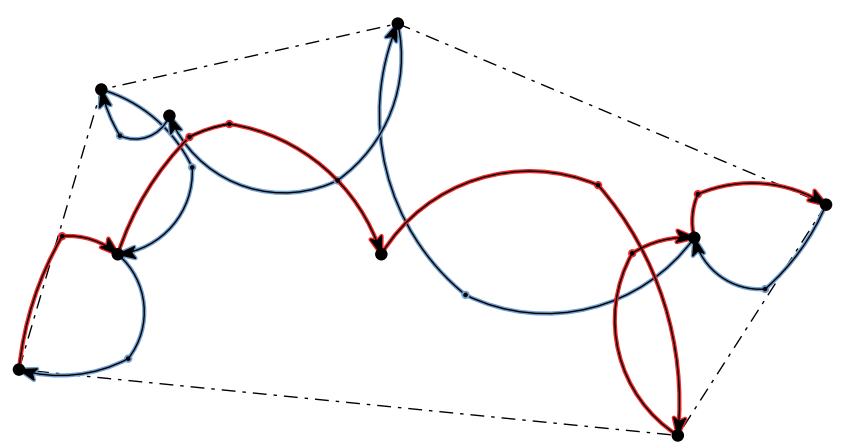

Figure 10: The colored chain of arcs of $P$.

3. Identify bichromatic intersecting links.

Note that the largest possible arc is a semicircle and, therefore, any arc can be partitioned into at most three segments to get a set of curves monotone with respect to an arbitrary direction. The arcs in $\mathcal{A}(P)$ can thus be transformed into a set $\mathcal{A}^{\prime}(P)$ of curves monotone with respect to the same direction. The Bentley and Ottmann plane sweep algorithm 9 ] 
can then be applied on $\mathcal{A}^{\prime}(P)$ to compute the intersection points between arcs. We discriminate from these points those belonging to bichromatic pairs of arcs. Pointers to the links containing the arcs involved in each intersection should be set up, so we can obtain the set of all bichromatic pairs of intersecting links in $\mathcal{A}(P)$.

4. Compute the sequence of overlap and release events.

Consider two extremal $\theta$-quadrants, denoted as $Q_{\theta}(p, q)$ and $Q_{\theta}(r, s)$, and a pair of arcs $\widehat{a b} \in C(p, q)$ and $\widehat{c d} \in C(r, s)$ (where $C(p, q)$ is the semicircle with diameter the segment $p q$, and $C(r, s)$ similarly) with their corresponding tracing intervals $\left(\alpha_{a}, \alpha_{b}\right)$ and $\left(\alpha_{c}, \alpha_{d}\right)$. See Figure 11. We say that $\widehat{a b}$ and $\widehat{c d}$ admit overlapping $\theta$-quadrants, if $Q_{\varphi}(p, q)$ and $Q_{\psi}(r, s)$ overlap for some $\varphi \in\left(\alpha_{a}, \alpha_{b}\right)$ and $\psi \in\left(\alpha_{c}, \alpha_{d}\right)$.

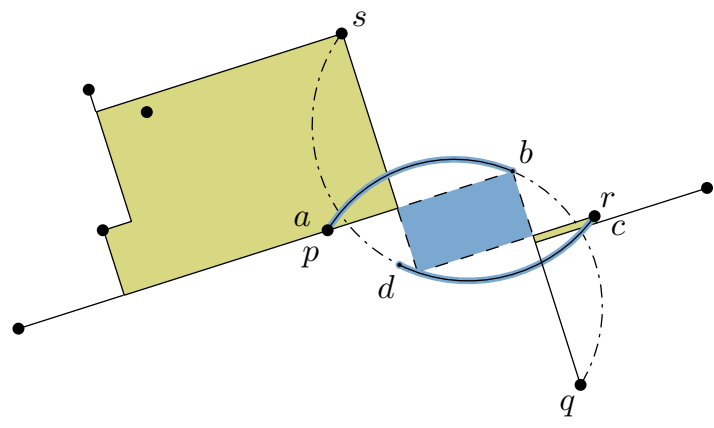

(a)

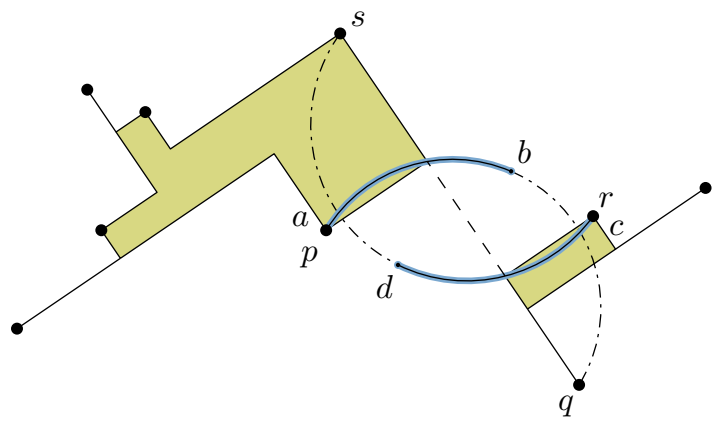

(b)

Figure 11: (a) The arcs $\widehat{a b}$ and $\widehat{c d}$ (highlighted) admit overlapping $\theta$-quadrants. (b) Release event of the corresponding overlapping region.

Assume that $\widehat{a b}$ and $\widehat{c d}$ admit overlapping of opposite $\theta$-quadrants (recall Lemma 1) and, without loss of generality, suppose that $p$ precedes $q$ in $\mathcal{V}_{\theta}(P)$ for all $\theta \in\left(\alpha_{a}, \alpha_{b}\right)$, and that $r$ precedes $s$ for all $\theta \in\left(\alpha_{c}, \alpha_{d}\right)$. It is not hard to see that, since the extremal $\theta$-quadrants $Q_{\theta}(p, q)$ and $Q_{\theta}(r, s)$ are opposite to each other, $\left(\alpha_{a}, \alpha_{b}\right) \cap\left(\alpha_{c}+\pi, \alpha_{d}+\pi\right)$ is not empty and, during this interval, the ray of $Q_{\theta}(p, q)$ passing through $p$ (resp. $q$ ) is parallel to the ray of $Q_{\theta}(r, s)$ passing through $r$ (resp. $s$ ). Note that $q$ and $s$ lie on different sides of the line $\ell_{p, r}$ passing through $p$ and $r$, as otherwise $Q_{\theta}(p, q) \cap Q_{\theta}(r, s)$ could not be $P$-free. For the same reason, the points $p, r$ lie on opposite sides of $\ell_{q, s}$ and, therefore, the line segments $\overline{p r}$ and $\overline{q s}$ intersect with each other. It is easy to see that this intersection is contained in the overlapping region generated by $Q_{\theta}(p, q)$ and $Q_{\theta}(r, s)$ and, thus, we have that $\overline{p r} \cap \overline{q s} \subset D(p, q) \cap D(r, s)$. Note that the angular interval of maximum size where $Q_{\theta}(p, q)$ and $Q_{\theta}(r, s)$ may overlap, called the maximum overlapping interval, is bounded by the orientations where $x_{\theta}$ is parallel to $\overline{p r}$ and where $y_{\theta}$ is parallel to $\overline{q s}$.

Observation 1. The arcs $\widehat{a b}$ and $\widehat{c d}$ admit overlapping $\theta$-quadrants if, and only if, $Q_{\theta}(p, q)$ and $Q_{\theta}(r, s)$ define a maximum overlapping interval $\left(\theta_{1}, \theta_{2}\right)$, and

$$
\left(\theta_{1}, \theta_{2}\right) \cap\left(\alpha_{a}, \alpha_{b}\right) \cap\left(\alpha_{c}+\pi, \alpha_{d}+\pi\right) \neq \emptyset .
$$

Let $\left\langle\widehat{a_{1} a_{2}}, \widehat{a_{2} a_{3}}, \ldots, \widehat{a_{k} a_{k+1}}\right\rangle$ be the set of arcs for all $\theta \in[0,2 \pi)$, where $k=O(n)$, labeled while traversing $\mathcal{A}(P)$ in such way that the vertices of $\partial(\mathcal{C H}(P))$ are visited in counterclockwise circular order. We denote with $\widehat{\ell}_{u, v}$ the subsequence $\left\langle\left(a_{u}, a_{u+1}\right), \ldots,\left(a_{v}, a_{v+1}\right)\right\rangle$ of consecutive arcs in $\mathcal{A}(P)$ forming a link. Note that the extremal intervals of the arcs in $\hat{\ell}_{u, v}$ define the sequence $\left\langle\alpha_{a_{u}}, \ldots, \alpha_{a_{v+1}}\right\rangle$ of increasing angles. See Figure 12 . 


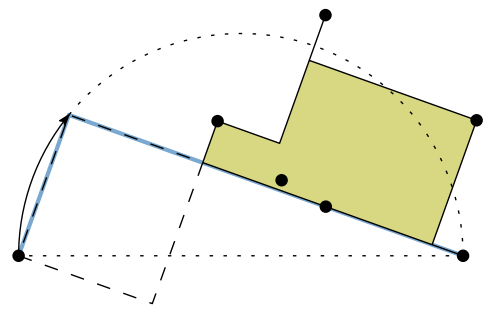

(a)

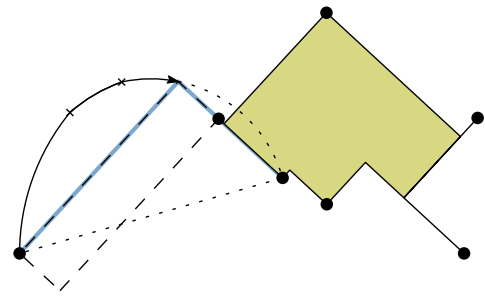

(c)

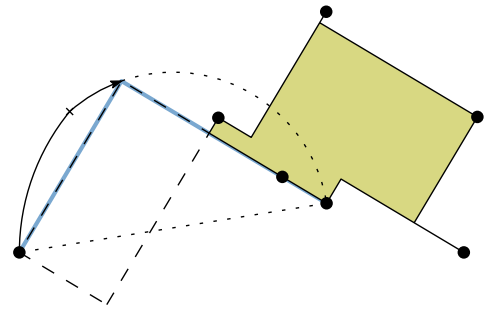

(b)

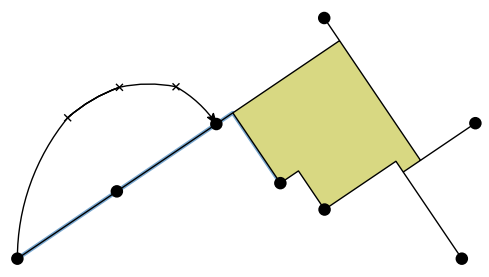

(d)

Figure 12: The extremal intervals in the same link define a sequence of increasing angles.

Based on the Observation 1 above, we can compute the overlapping regions generated by the arcs belonging to a pair $\hat{\ell}_{u, v}$ and $\hat{\ell}_{s, t}$ of intersecting links, as an extension of the well-known linear-time merge procedure that operates on the lists $\left\langle\alpha_{a_{u}}, \ldots, \alpha_{a_{v+1}}\right\rangle$ and $\left\langle\alpha_{a_{s}}+\pi, \ldots, \alpha_{a_{t+1}}+\pi\right\rangle$, and their corresponding arc sequences: the intersection between a pair of non-consecutive maximal intervals in the merged list is empty. These pairs can be ignored, as they do not comply with Observation 1 and, therefore, at most a linear number of pairs of $\operatorname{arcs}$ in $\hat{\ell}_{u, v}$ and $\hat{\ell}_{s, t}$ admit overlapping $\theta$-quadrants.

Let $\ell_{u, v}$ and $\ell_{s, t}$ be two intersecting links containing respectively, $n_{u, v}=u-v+1$ and $n_{s, t}=t-s+1$ arcs. At most $O\left(n_{u, v}+n_{s, t}\right)$ pairs of arcs admit overlapping $\theta$-quadrants. The overlapping regions generated by the admitted extremal $\theta$-quadrants can be computed using $O\left(n_{u, v}+n_{s, t}\right)$ time and space.

By Theorem 2, we know that Step 1 takes $O(n \log n)$ time and $O(n)$ space, as a constant number of additional operations are performed at each event while traversing the vertex event sequence. Step 2 takes $O(n)$ time and space, as the number of arcs in $\mathcal{A}(P)$ is linear in the number of elements in $P$. To compute $\mathcal{A}(P)$ we require a linear run on $\mathcal{A}(P)$ and, by Theorem 2 , the Bentley and Ottmann [9] plane sweep processes $\mathcal{A}(P)$ in $O(n \log n)$ time and $O(n)$ space. Additional linear time is needed to discriminate, from the resulting intersection points, those belonging to bichromatic intersecting links, thus Step 3 requires a total of $O(n \log n)$ time and $O(n)$ space. Finally, from Lemma 4 and Theorem 2 , and the facts that there is a linear number of arcs and each arc belongs to a single link, Step 4 requires $O(n \log n)$ time and $O(n)$ space. Therefore, we have the following result:

Theorem 3. The sequence of overlap and release events of $\mathcal{R H}_{\theta}(P)$, while $\theta$ increases from 0 to $2 \pi$, can be computed in $O(n \log n)$ time and $O(n)$ space.

\subsubsection{Sweeping the sequence of overlap and release events}

We now store $\mathcal{S}_{\theta}(P)$ in a hash table, using as keys tuples with the points supporting the overlapping $\theta$-quadrants, in the same order as they are found while traversing $\mathcal{V}_{\theta}(P)$. For an example, the overlapping region in Figure 11(b) would be stored in $\mathcal{S}_{\theta}(P)$ using as key the tuple $(p, q, r, s)$. 

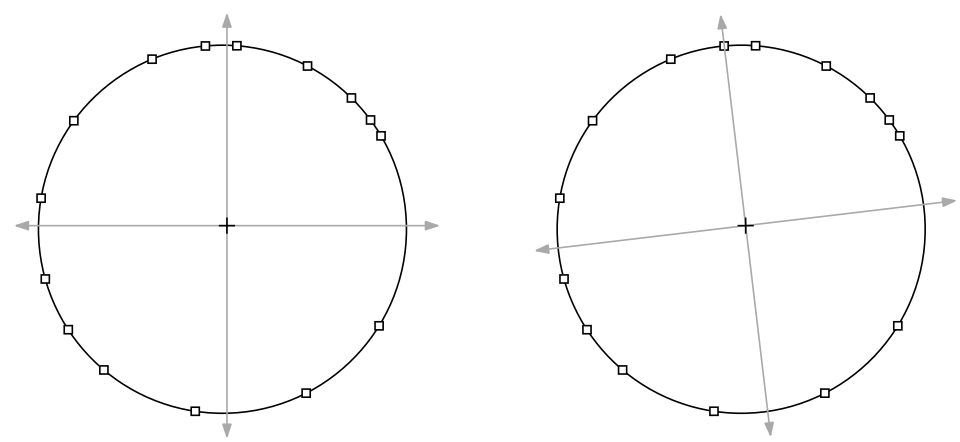

Figure 13: Left: Representation of overlap and release events and 0-quadrants. Right: Simultaneous rotation of the four $\theta$-quadrants, stopping when one of their defining rays passes over an overlap event.

We further store the sequence of overlap and release events as points on an unit circle $[0,2 \pi)$, over which we represent the $\theta$-quadrants. See Figure 13 , left. Then, we counterclockwise rotate the four $\theta$-quadrants simultaneously around the center, stopping when one of their defining rays passes over an overlap event, in order to update $\mathcal{S}_{\theta}(P)$ accordingly. See Figure 13 , right. It is easy to see that, at any fixed value of $\theta$ there are $O(n)$ overlapping regions in $\mathcal{R \mathcal { H }}_{\theta}(P)$, which can be computed in linear time from $\mathcal{V}_{\theta}(P)$. Since, by Theorem 2 , there are $O(n)$ overlap and release events, we obtain the following result.

Theorem 4. Using the sequence of overlap and release events, the set $\mathcal{S}_{\theta}(P)$ can be maintained while $\theta$ increases from 0 to $2 \pi$ in $O(n)$ time and $O(n)$ space.

\subsection{Minimum area}

In this section we adapt the results from Bae et al. 8 , to compute the value of $\theta$ that minimizes the area of $\mathcal{R H}_{\theta}(P)$ in optimal $O(n \log n)$ time and $O(n)$ space.

Let $(\alpha, \beta)$ be an angular interval in $[0,2 \pi)$ containing no events. Extending Equation (2), we express the area of $\mathcal{R} \mathcal{H}_{\theta}(P)$ for any $\theta \in(\alpha, \beta)$ as

$$
\operatorname{area}\left(\mathcal{R H}_{\theta}(P)\right)=\operatorname{area}(\mathcal{P}(\theta))-\sum_{j} \operatorname{area}\left(\triangle_{j}(\theta)\right)+\sum_{k} \operatorname{area}\left(\square_{k}(\theta)\right) .
$$

Remember that $\mathcal{P}(\theta)$ denotes the polygon having the points in $\mathcal{V}_{\theta}(P)$ as vertices, with an edge connecting two vertices if they are consecutive elements in $\mathcal{V}_{\theta}(P)$. The term $\triangle_{j}(\theta)$ denotes the triangular region bounded by the line through two consecutive vertices $v_{j}, v_{j+1} \in \mathcal{V}_{\theta}(P)$, the line through $v_{j}$ parallel to the rotation of the $x$-axis, and the line through $v_{j+1}$ parallel to the rotation of the $y$-axis. Finally, the term $\square_{k}(\theta)$ denotes the $k$-th overlapping region in $\mathcal{S}_{\theta}(P)$. Recall Figure 3 .

We now show that at any particular value of $\theta$ we can evaluate Equation (3) in linear time and, as $\theta$ increases from 0 to $2 \pi$, a constant number of terms need to be updated at each event, regardless of its type.

\subsubsection{The polygon}

At any fixed value of $\theta$, the area of $\mathcal{P}(\theta)$ can be computed from $\mathcal{V}_{\theta}(P)$ in $O(n)$ time. The term area $(\mathcal{P}(\theta))$ changes only at vertex events. These changes can be handled in constant time: at an in (resp. out)-event, the area of a triangle needs to be subtracted (resp. added) to the previous value of area $(\mathcal{P}(\theta))$. See Figure 14 . 


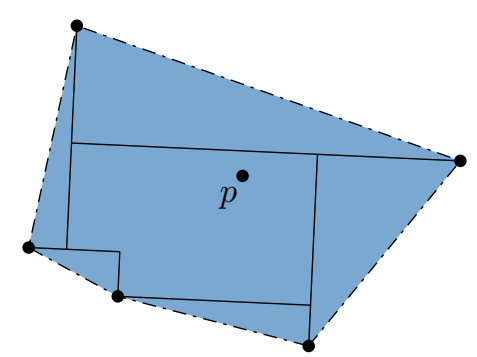

(a)

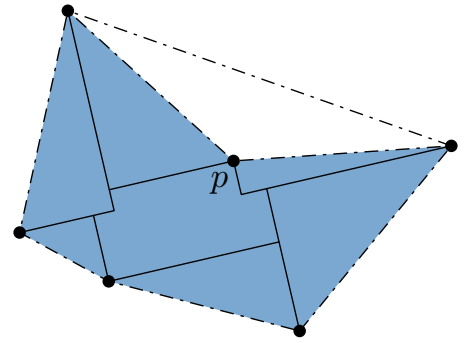

(b)

Figure 14: (a) The point $p$ is about to become a vertex. (b) After the insertion event of $p$, the area of the white triangle needs to be subtracted from area $(\mathcal{P}(\theta))$.

\subsubsection{The triangular regions}

According to Bae et al. [8], the area of $\triangle_{j}(\theta)$ can be expressed as

$$
\operatorname{area}\left(\triangle_{j}(\theta)\right)=b_{j}^{2} \cdot \cos \left(c_{j}+(\theta-\alpha)\right) \cdot \sin \left(c_{j}+(\theta-\alpha)\right),
$$

where $b_{j}^{2}$ and $c_{j}$ are constant values depending on the coordinates of the vertices supporting the $\theta$-quadrant which bounds $\triangle_{j}(\theta)$. Contracting Equation (4) we have that

$$
\begin{aligned}
\operatorname{area}\left(\triangle_{j}(\theta)\right) & =\frac{1}{2} b_{j}^{2} \cdot \sin 2\left(c_{j}+(\theta-\alpha)\right) \\
& =\frac{1}{2} b_{j}^{2} \cdot\left[\sin \left(2 c_{j}\right) \cdot \cos 2(\theta-\alpha)+\cos \left(2 c_{j}\right) \cdot \sin 2(\theta-\alpha)\right] \\
& =B_{j} \cdot \cos 2(\theta-\alpha)+C_{j} \cdot \sin 2(\theta-\alpha),
\end{aligned}
$$

where $B_{j}=\frac{1}{2} b_{j}^{2} \cdot \sin \left(2 c_{j}\right)$ and $C_{j}=\frac{1}{2} b_{j}^{2} \cdot \cos \left(2 c_{j}\right)$. Equation 5 can be computed in constant time, and there are $O(n)$ triangles, since the number of vertices in $\mathcal{V}_{\theta}(P)$ is linear. Thus, at any fixed value of $\theta$ the term $\sum_{j}$ area $\left(\triangle_{j}(\theta)\right)$ can be computed in $O(n)$ time. At an insertion event the term for one triangle is removed from $\sum_{j}$ area $\left(\triangle_{j}(\theta)\right)$ and, as a vertex supports at most two extremal $\theta$-quadrants, the terms of at most two triangles are added. The converse occurs for deletion events. The term $\sum_{j} \operatorname{area}\left(\triangle_{j}(\theta)\right)$ is not affected by overlap or release events. See Figure 15 .

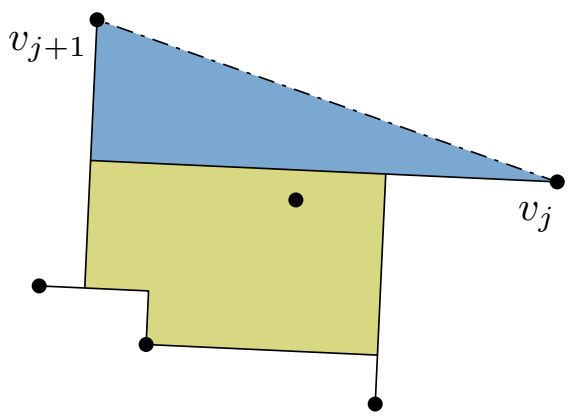

(a)

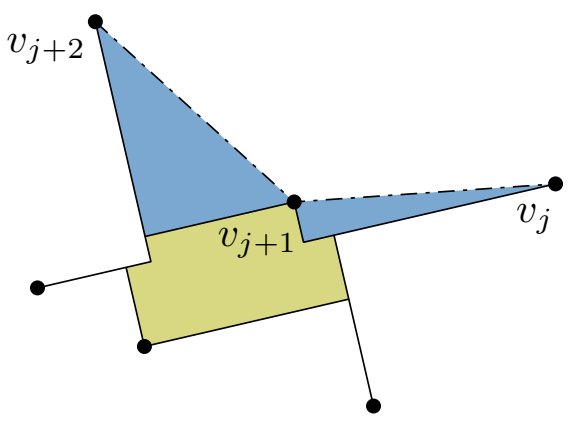

(b)

Figure 15: Updating the term $\sum_{j} \operatorname{area}\left(\triangle_{j}(\theta)\right)$. At an insertion event, (a) at most one triangle have to be removed, and (b) at most two triangles have to be added. 


\subsubsection{The overlapping regions}

According to Bae et al. [8] the area of the $k$-th overlapping region can be expressed as

$$
\begin{aligned}
\operatorname{area}\left(\square_{k}(\theta)\right) & =\left|\left(x_{i}-x_{j}\right) \cos (\theta-\alpha)+\left(y_{i}-y_{j}\right) \sin (\theta-\alpha)\right| \\
& \times\left|\left(y_{i+1}-y_{j+1}\right) \cos (\theta-\alpha)+\left(y_{i+1}-y_{j+i}\right) \sin (\theta-\alpha)\right|,
\end{aligned}
$$

where $\left(x_{i}, y_{i}\right)$ are the coordinates of the vertex $p_{i}$, and similarly for $p_{i}, p_{i+1}, p_{j}$, and $p_{j+1}$. These four points are the vertices that support the overlapping $\theta$-quadrants that generate $\square_{k}(\theta)$. Contracting Equation 6 the area of the $k$-th overlapping region can be expressed as

$$
\operatorname{area}\left(\square_{k}(\theta)\right)=B_{k}+C_{k} \cos 2(\theta-\alpha)+D_{k} \sin 2(\theta-\alpha),
$$

where $B_{k}, C_{k}$, and $D_{k}$ are constants depending on the coordinates of the vertices supporting the overlapping $\theta$-quadrants that generate $\square_{k}(\theta)$. Equation $(7)$ can be computed in constant time and there are $O(n)$ overlapping regions in $\mathcal{S}_{\theta}(P)$, so at any fixed value of $\theta$ the term $\sum_{k}$ area $\left(\square_{k}(\theta)\right)$ can be computed in $O(n)$ time. Overlap or release events require the term of a single overlapping region to be added to or deleted from $\sum_{k}$ area $\left(\square_{k}(\theta)\right)$. As a vertex supports at most two extremal $\theta$-quadrants, at an overlap event the terms of a constant number of overlapping regions are added or deleted.

Before describing the minimum area algorithm, we need the next three important properties of area $\left(\mathcal{R} \mathcal{H}_{\theta}(P)\right)$. First of all, from Lemma 4 in Bae et al. [8], there are configurations of points such that the optimal area does not occur at in- or out vertex events, i.e., the value of $\theta$ for which area $\left(\mathcal{R} \mathcal{H}_{\theta}(P)\right)$ is minimum in $(\alpha, \beta)$ will be a value such that $\alpha<\theta<\beta$. Second, Equation (3) has $O(n)$ terms for any $\theta \in(\alpha, \beta)$ and thus, it can be reduced to

$$
\operatorname{area}\left(\mathcal{R H}_{\theta}(P)\right)=C+D \cos 2(\theta-\alpha)+E \sin 2(\theta-\alpha)
$$

in $O(n)$ time. The terms $C, D$ and $E$ denote constants resulting from adding up the constant values in area $(\mathcal{P}(\theta))$ and in Equations (5) and (7). Finally, as Equation (8) has a constant number of inflection points in $[0,2 \pi)$, a constant number of operations suffice to obtain the value of $\theta$ that minimizes area $\left(\mathcal{O H}_{\theta}(P)\right)$ in $(\alpha, \beta)$. Notice that at this point we can also ask for the value of $\theta$ that maximizes area $\left(\mathcal{O H}_{\theta}(P)\right)$ in $(\alpha, \beta)$, and that, in fact, the maximum can be also take place in any of the extremes $\alpha$ or $\beta$.

\subsubsection{The search algorithm}

We now outline the algorithm to compute the angle $\theta$ for which $\mathcal{R} \mathcal{H}_{\theta}(P)$ has minimum area.

\section{Compute the sequence of events.}

Compute the sequence of vertex in- and out-events, as described in Subsection 2.1, and the sequence of overlap and release events, as described in Subsection 2.2.4. Merge both sequences into a single circular sequence of angles $\left\langle\theta_{1}, \theta_{2}, \ldots, \theta_{m-1}, \theta_{m}, \theta_{1}\right\rangle, m \in O(n)$, which we can represent in a circular table as in Figure 13. Clearly, while $\theta$ increases in $[0,2 \pi)$ the relevant features of $\mathcal{R H}_{\theta}(P)$ remain unchanged during each interval $\left(\theta_{i}, \theta_{i+1}\right)$, and each angle $\theta_{i}$ is an in-, out-, overlap-, or release-event.

\section{Initialize the angular sweep.}

Represent the four $\theta$-quadrants over the circular table, as we did in Figure 13 . Without loss of generality, assume that the first (counterclockwise) defining ray of the first $\theta$-quadrant 
intersects the angular interval $\left(\theta_{1}, \theta_{2}\right)$. Compute the sets $\mathcal{V}_{\theta_{1}}$ and $\mathcal{S}_{\theta_{1}}$ for the current $\theta_{1}$ as before, and express area $\left(\mathcal{R} \mathcal{H}_{\theta}(P)\right)$ for $\theta \in\left[\theta_{1}, \theta_{2}\right)$ using Equation (8). Compute the constant values in this equation considering the restriction $\theta \in\left[\theta_{1}, \theta_{2}\right)$. Optimize the resulting equation to compute the angle $\theta_{\min }$ (resp. $\theta_{\max }$ ) of minimum (resp. maximum) area.

\section{Perform the angular sweep.}

Rotate simultaneously the four four $\theta$-quadrants, as we did in Subsection 2.2.5. During the sweeping process, update $\mathcal{V}_{\theta}(P)$ and $\mathcal{S}_{\theta}(P)$ as explained before. Additionally, at each event:

(a) Update Equation (8) by adding or subtracting terms as previously explained.

(b) Optimize the updated version of Equation (8) to obtain the local angle of minimum (resp. maximum) area, and replace $\theta_{\min }\left(\right.$ resp. $\left.\theta_{\max }\right)$ if area $\left(\mathcal{R} \mathcal{H}_{\theta}(P)\right)$ is improved.

From Theorems 1 and 3 , computing the sequences of vertex and overlap and release events takes $O(n \log n)$ time and $O(n)$ space. As both sequences have $O(n)$ events, we require linear time to merge them into the sequence of events and thus, Step 1 consumes a total of $O(n \log n)$ time and $O(n)$ space. At Step 2, $\mathcal{V}_{\theta}(P)$ can be computed in $O(n \log n)$ time and $O(n)$ space (see [17]), and $\mathcal{S}_{\theta}(P)$ can be easily computed from $\mathcal{V}_{\theta}(P)$ in linear time. An additional linear time is required to obtain Equation (8), while $\theta_{\min }$ (resp. $\theta_{\max }$ ) can be computed in constant time. This gives a total of $O(n \log n)$ time and $O(n)$ space. Finally, by Theorems 1 and 4 . respectively, maintaining $\mathcal{V}_{\theta}(P)$ and $\mathcal{S}_{\theta}(P)$ requires $O(n \log n)$ time and linear space for each. Step 3(a) and Step 3(b) are repeated $O(n)$ times (one per event in the sequence) and, as we described before, each repetition takes constant time. Therefore, to perform Step 3 we consume a total of $O(n \log n)$ time and $O(n)$ space. Notice that, after the sweeping process is finished, in additional $O(n \log n)$ time and $O(n)$ space we can compute both $\mathcal{R H}_{\theta}(P)$ and $\operatorname{area}\left(\mathcal{R H}_{\theta}(P)\right)$ for the angle $\theta_{\min }$ (resp. $\theta_{\max }$ ) giving the minimum (resp. maximum) area.

The optimality of the algorithm is clear since given $\mathcal{R H}_{\theta}(P)$ we can compute in linear time $\mathcal{C H}\left(\mathcal{R H}_{\theta}(P)\right)=\mathcal{C H}(P)$, and it is known that computing the convex hull of a set of $n$ points in the plane has an $\Omega(n \log n)$ time lower bound [23]. From this analysis, we obtain our main result.

Theorem 5. Computing $\mathcal{R H}_{\theta}(P)$ for the value of $\theta \in[0,2 \pi)$ such that $\mathcal{R H}_{\theta}(P)$ has minimum (or maximum) area can be done in optimal $O(n \log n)$ time and $O(n)$ space.

\section{$3 \quad \mathcal{O}$-hull of a point set}

As mentioned in Section 1, orthogonal convexity can be generalized to consider a finite set $\mathcal{O}$ of $k$ different lines passing through the origin. A set is thus said to be $\mathcal{O}$-convex if its intersection with any line parallel to an element of $\mathcal{O}$ is either connected or empty. Following the lines in Section 2, here we study the $\mathcal{O}$-convex hull of a set $P$ of $n$ points.

\subsection{Definitions}

Let us label the lines in $\mathcal{O}$ as $\ell_{1}, \ldots, \ell_{k}$, so that $i<j$ implies that the slope of $\ell_{i}$ is smaller than the slope of $\ell_{j}$. We consider the set of lines in $\mathcal{O}$ being sorted by slope; otherwise we would spend $O(k \log k)$ time for that sorting and add this complexity to the total complexity of the upcoming algorithms. Nevertheless, the number $k$ of lines will be smaller than the number $n$ of input points of the problem because if $k>n$ then, at most $n$ lines can participate as it 
will becomes clear in Subsection 3.2.1, and thus $O(k \log k)$ will be in $O(n \log n)$. However, the complexity of some of our algorithms can be sensitive to $k$, which will appear as a multiplicative factor.

The origin splits each $\ell_{i}$ into two rays $r_{i}$ and $r_{i+k}$, generating a set of $2 k$ rays. Hereinafter, indices are such that $2 k+i:=i$. Given two indexes $i$ and $j$, we define the wedge $W_{i, j}$ to be the open region spanned as we rotate $r_{i}$ in the counterclockwise direction until it reaches $r_{j}$. A translation of a $W_{i, j}$ wedge will be called a $W_{i}^{j}$ wedge, and one of these will be said to be $P$-free if it does not contain any point of $P$. Of particular interest to us is the set of $W_{i+1}^{i+k}$ wedges, $i=1, \ldots, 2 k$, which we will call $\mathcal{O}$-wedges for short. See Figure 16 .
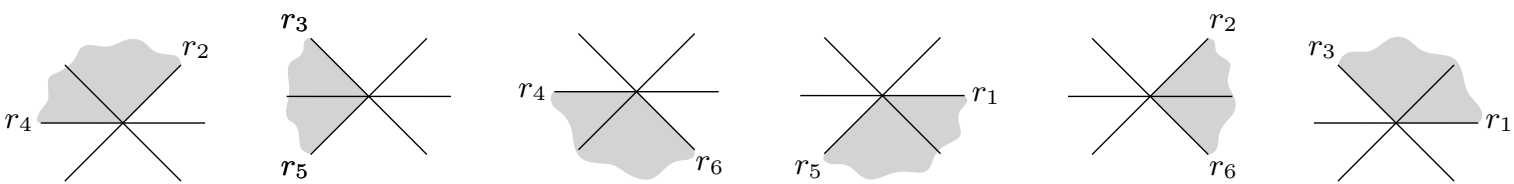

Figure 16: Top: A set $\mathcal{O}$ with $k=3$. Bottom: From left to right, the corresponding $W_{i+1, i+k}$ wedges for $i=1, \ldots, 2 k$.

We denote by $\mathcal{W}^{i}$ the union of all the $P$-free $W_{i+1}^{i+k}$ wedges. Thus, by analogy with the orthogonal case in Section 2 , the $\mathcal{O}$-hull of $P$ is (see Figure 17 for an example):

$$
\mathcal{O H}(P)=\mathbb{R}^{2} \backslash \bigcup_{i=1}^{2 k} \mathcal{W}^{i} .
$$
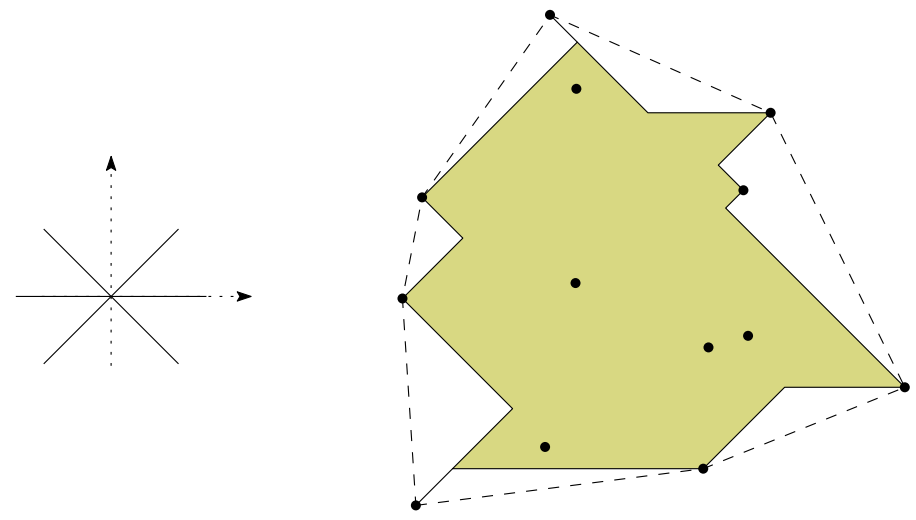

Figure 17: The set $\mathcal{O}$ in Figure 16 and the $\mathcal{O}$-hull $\mathcal{O H}(P)$ for a point set $P$.

As in Section 2, we consider rotations of the axes by an angle $\theta$. Let $\mathcal{O}_{\theta}$ be the set of lines obtained after rotating the elements of $\mathcal{O}$ by an angle $\theta$. Clearly, the $\mathcal{O}_{\theta}$-hull of $P$, denoted as $\mathcal{O H}_{\theta}(P)$, changes while $\theta$ goes from 0 to $2 \pi$, since rotating $\mathcal{O}$ by an angle $\theta$ makes the wedges $W_{i, j}$ rotate as well and, hence, the sets $\mathcal{W}^{i}$ change accordingly. We will denote the resulting set as $\mathcal{W}_{\theta}^{i}$, so that $\mathcal{O H}_{\theta}(P)$ is now defined as (see Figure 18 for an example):

$$
\mathcal{O H}_{\theta}(P)=\mathbb{R}^{2} \backslash \bigcup_{i=1}^{2 k} \mathcal{W}_{\theta}^{i}
$$



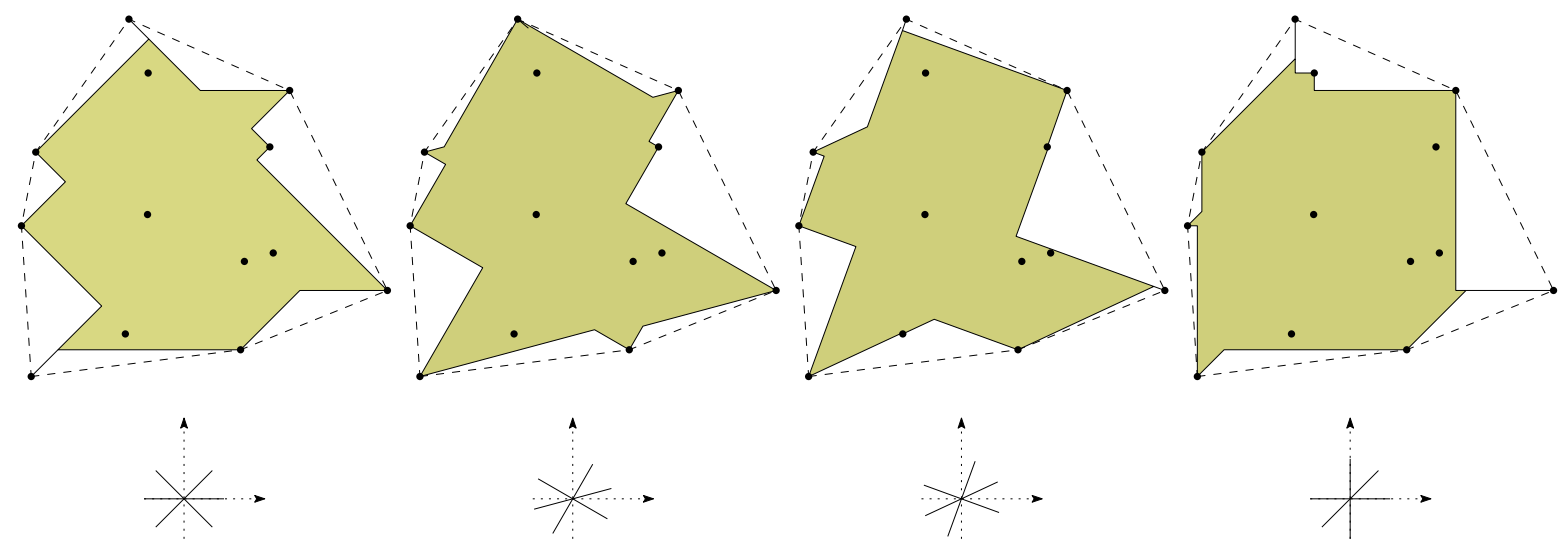

Figure 18: Changes on the $\mathcal{O}_{\theta}$-hull $\mathcal{O H}_{\theta}(P)$ while changing $\theta$.

\subsection{Computing $\mathcal{O H}(P)$}

In this subsection we provide an optimal $O(n \log n)$ time and $O(n)$ space algorithm to compute the $\mathcal{O}$-hull $\mathcal{O H}(P)$ of a set $P$ of $n$ points.

\subsubsection{Computing the vertices}

For each $r_{i}$, compute first the directed line parallel to $r_{i}$ which supports (the pre-computed) $\mathcal{C H}(P)$ leaving $P$ on its right side. Suppose, without loss of generality, that each of these lines intersects $\mathcal{C H}(P)$ at a single point, labelled $p_{s_{i}}, i=1, \ldots, 2 k$. Notice that it is not necessarily true that $p_{s_{i}}$ is different from $p_{s_{i+1}}$. Thus, $p_{s_{1}}, p_{s_{2}}, \ldots, p_{s_{2 k}}$ are vertices of the boundary of the $\mathcal{O}$-hull, $\partial(\mathcal{O H}(P))$, see Figure 19, labelled as we meet them in counterclockwise order. Note also that these $p_{s_{i}}$ might not give all the vertices of $\partial(\mathcal{O H}(P))$, see again Figure 19 .

Because of the definition $\mathcal{O H}(P)=\mathbb{R}^{2} \backslash \cup_{i=1}^{2 k} \mathcal{W}^{i}$, we need to compute $\partial\left(\mathcal{W}^{i}\right)$ and this requires knowing when a wedge in $\mathcal{W}^{i}$ can intersect the interior of $\mathcal{C H}(P)$. It is easy to see that there are wedges in $\mathcal{W}^{i}$ that intersect the interior of $\mathcal{C H}(P)$ if, and only if, $p_{s_{i}} \neq p_{s_{i+1}}$, and that any wedge in $\mathcal{W}^{i}$ intersecting the interior of $\mathcal{C H}(P)$ necessarily does so by intersecting an edge of $\partial(\mathcal{C H}(P))$ whose endpoints $p_{j}, p_{j+1}$ fulfill $s_{i} \leq j, j+1<s_{i+1}$. See Figure 19 . Abusing the notation, let us denote by $\left[s_{i}, s_{i+1}\right]$ the closed interval of those indices of vertices on $\partial(\mathcal{C H}(P))$ between $s_{i}$ and $s_{i+1}$, called the stabbing interval of $\mathcal{W}^{i}$. See the caption of Figure 19.

Observation 2. If $s$ belongs to the stabbing interval $\left[s_{i}, s_{i+1}\right]$ of a wedge in $\mathcal{W}^{i}$, then the orientation of the edge $e_{s}$ of $\partial(\mathcal{C H}(P))$ belongs to the sector formed by the rays $r_{i}$ and $r_{i+1}$ in $\mathcal{O}$. See Figure 19 once more. Also note that, if $\mathcal{O}$ contains the supporting lines of the $h$ edges in $\partial(\mathcal{C H}(P))$, then the stabbing interval of each of all the $\mathcal{W}^{i}$ is a point and therefore $\mathcal{O H}(P)=\mathcal{C H}(P)$.

It is easy to see that we can calculate the elements $p_{s_{1}}, \ldots, p_{s_{2 k}}$ on $\partial(\mathcal{C H}(P))$ in $O(n \log n)$ time, in fact, in $O(k \log n)$ time. This gives us the endpoints of the stabbing interval $\left[s_{i}, s_{i+1}\right]$. Only those intervals not being a single index will be needed, the others can be discarded. Next, we calculate the alternating polygonal chain on $\partial(\mathcal{O H}(P))$ connecting $p_{s_{i}}$ to $p_{s_{i+1}}$, which we refer to as staircase. 

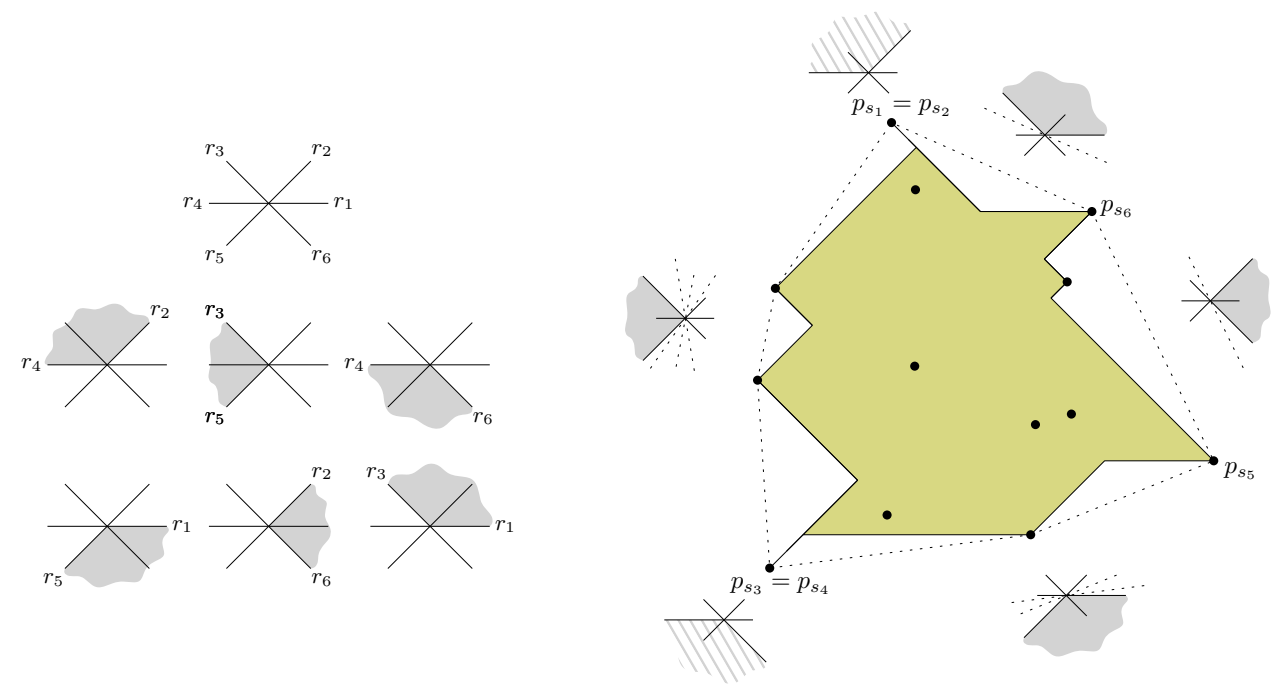

Figure 19: Left: Recalling Figure 16. Right: The $\mathcal{O}$-hull $\mathcal{O H}(P)$ in Figure 17, showing which edges of $\partial(\mathcal{C H}(P))$, if any, are intersected by wedges in each $\mathcal{W}^{i}$. Note that wedges in $\mathcal{W}^{1}$ and $\mathcal{W}^{3}$, for which examples are lined instead of solid, do not intersect the interior of $\mathcal{C H}(P)$. For $p_{1}$ being the uppermost point and labelling counterclockwise the vertices of $\partial(\mathcal{C H}(P))$, the stabbing intervals are $[1,4],[4,6],[6,7]$, and $[7,1]$.

\subsubsection{Computing the staircases}

The staircase connecting $p_{s_{i}}$ to $p_{s_{i+1}}$ is determined by wedges in $\mathcal{W}^{i}$ and is contained in the boundary $\partial\left(\mathcal{W}^{i}\right)$. Counterclockwise around $\mathcal{O H}(P)$, right turns arise at apexes of wedges in $\mathcal{W}^{i}$ which we call extremal, and left turns arise at points of $P$ which we call the supporting points of those extremal wedges. See Figure 20.
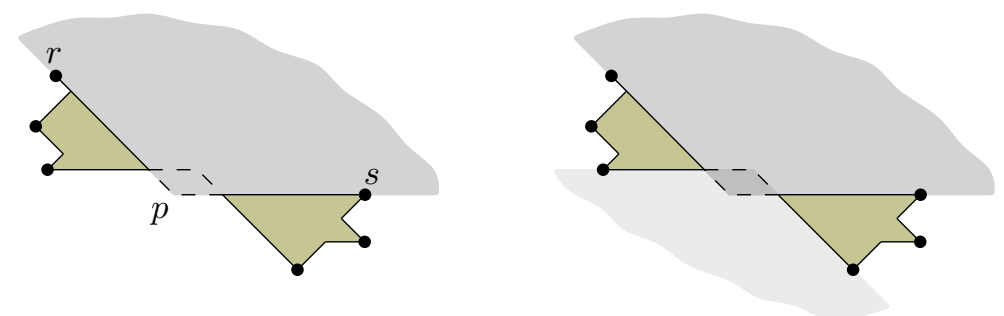

Figure 20: Another $\mathcal{O H}_{\theta}(P)$ for the $\mathcal{O}$ in Figure 16. Left: An extremal wedge in $\mathcal{W}^{6}$ with apex $p$ and supporting points $r$ and $s$. Right: Opposite extremal $\mathcal{O}$-wedges, one in $\mathcal{W}^{6}$ and the other in $\mathcal{W}^{3}$. In dark, the overlapping region, which is now a rhomboid instead of a rectangle.

Before presenting how to compute the staircase, let us note that $\mathcal{O H}(P)$ can also be disconnected. Similarly to Subsection 2.2.1, we say that a pair of extremal wedges are opposite to each other if one of them is in $\mathcal{W}^{i}$ and the other in $\mathcal{W}^{i+k}$ (i.e., one is defined by parallels to the rays $r_{i+1}$ and $r_{i+k}$ and the other by parallels to the rays $r_{i+k+1}$ and $r_{i}$ ). As seen in Figure 20, a non-empty intersection between two opposite $\mathcal{O}$-wedges results in $\mathcal{O H}(P)$ being disconnected. In such case, we say that the intersecting wedges overlap, and refer to their intersection as their overlapping region. The following lemma is a straightforward generalization of Lemma 1.

Lemma 5. If two extremal $\mathcal{O}$-wedges have nonempty intersection, then they have to be opposite. When this happens, $\mathcal{O H}_{\theta}(P)$ gets disconnected. 
We now proceed with the computation of the staircases, starting by the computation of their supporting points. In order to do so, we make use of an algorithm by Avis et al. 77. Let us call $\alpha_{i}$ the angle defined by two consecutive rays $r_{i}$ and $r_{i+1}$, so that the wedges in $\mathcal{W}^{i}$ have aperture angle $\Theta_{i}=\pi-\alpha_{i}$. We consider $\Theta=\min \left\{\Theta_{i}: i=1, \ldots, 2 k\right\}$ and we will distinguish two cases: either $\Theta \geq \frac{\pi}{2}$ or $\Theta<\frac{\pi}{2}$ according to the "well distributed" criteria of the lines in $\mathcal{O}$.

For the first case where $\Theta \geq \frac{\pi}{2}$, i.e., all the sectors defined by the $k$ lines have aperture angle at most $\frac{\pi}{2}$, the algorithm by Avis et al. [7 finds, in $O(n \log n)$ time and $O(n)$ space, the maximal $\Theta$-escaping wedges, defined as those (i) having as apex a point $p \in P$, (ii) having aperture angle at least $\Theta$, and (iii) being $P$-free, i.e., not containing any point of $P$ in its interior. (In other words, maximal $\Theta$-escaping wedges are the maximal wedges allowing an angle $\Theta$ to escape from $p$ without hitting other points of $P$.) The points being apexes of a maximal $\Theta$-escaping wedge are called $\Theta$-maxima. Indeed, for each such wedge the algorithm by Avis et al. [7] provides its two defining rays. Thus, the algorithm gives at most three maximal $\Theta$-escaping intervals for every $p \in P$, hence a linear number in total.
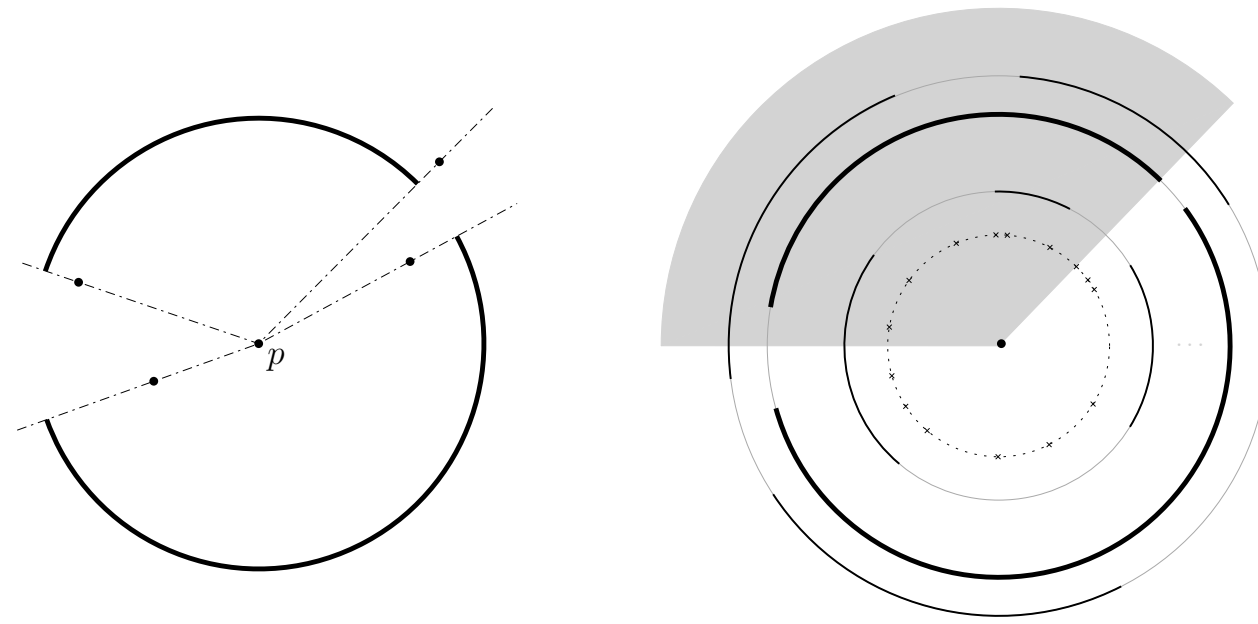

Figure 21: Left: Escaping intervals for a point $p$ and $\Theta=\frac{\pi}{2}$ as in Figure 16. Right: Circular table where the solid circles correspond to the points $p_{1}, \ldots, p_{n}$ from the inside to the outside. On them, the $\Theta$-escaping intervals, where the ones for the $p$ depicted in the left are highlighted. In gray, the stabbing interval corresponding to the wedge $W_{2,4}$ from Figure 16 (the other stabbing intervals are omitted for the sake of clarity). Finally, the innermost circle reflects, as small marks, the vertex events in $[0,2 \pi)$ corresponding to the endpoints of the escaping intervals.

We will store these intervals in a circular table, together with the stabbing wedges $W_{i+1, i+k}$. See Figure 21. Doing so, when a stabbing wedge $W_{i+1, i+k}$ fits into the escaping interval of a point $p$, we know that $p$ is not only a $\Theta$-maxima, but actually a $\Theta_{i}$-maxima, which is indeed equivalent to be a supporting point in $\partial\left(\mathcal{W}^{i}\right)$. (In Figure 21, right, the gray stabbing interval from the wedge $W_{2,4}$ does not fit into the black escaping interval, because in the left picture the wedge $W_{2}^{4}$ with apex $p$ cannot escape from $p$.)

Thus, in $O(n \log n)$ time and $O(n)$ space we can sort the endpoints of the two types of intervals and sweep circularly the table, stopping at the defining rays of the wedges $W_{i+1, i+k}$ to check if the corresponding $p$ supports the staircase contained on $\partial\left(\mathcal{W}^{i}\right)$. This gives the set $\mathcal{V}(P)$ of vertices of $\mathcal{O H}(P)$. It just remains to obtain the boundary of $\mathcal{O H}(P)$, for which standard techniques [23] can be used in order to compute the staircases $\partial\left(\mathcal{W}^{i}\right)$ from their supporting points and to join them in $O(n \log n)$ time and $O(n)$ space. Hence, we have computed $\mathcal{O H}(P)$ in $O(n \log n)$ time and $O(n)$ space. This time complexity is optimal, since given $\mathcal{O H}(P)$ we can compute in linear time $\mathcal{C H}(\mathcal{O H}(P))=\mathcal{C H}(P)$, and it is known that computing the convex hull 
of a set of $n$ points in the plane has an $\Omega(n \log n)$ time lower bound [23]. Therefore, we get the following result.

Theorem 6. Given a set $\mathcal{O}$ of $k$ lines such that $\Theta \geq \frac{\pi}{2}, \mathcal{O H}(P)$ can be computed in optimal $O(n \log n)$ time and $O(n)$ space.

For the second case where $\Theta<\frac{\pi}{2}$, the algorithm from Avis et al. [7] also works for $\Theta<\frac{\pi}{2}$, but now in $O\left(\frac{n}{\Theta} \log n\right)$ time and $O\left(\frac{n}{\Theta}\right)$ space. The algorithm gives at most $\frac{2 \pi}{\Theta}$ maximal $\Theta$-escaping intervals for every $p \in P$. Thus, we can construct a circular table as above, storing at most $\frac{2 \pi}{\Theta}$ circular intervals for each $p$, hence using $O\left(\frac{n}{\Theta}\right)$ space in total. Therefore, the previous result is extended as follows:

Theorem 7. Given a set $\mathcal{O}$ of $k$ lines such that $\Theta<\frac{\pi}{2}, \mathcal{O H}(P)$ can be computed in $O\left(\frac{n}{\Theta} \log n\right)$ time and $O\left(\frac{n}{\Theta}\right)$ space.

Note that the value $\frac{1}{\Theta}$ can be considered a constant for not too small values of $\Theta$.

\subsection{Computing and maintaining $\mathcal{O H}_{\theta}(P)$}

Recall that, as we rotate $\mathcal{O}$ by an angle $\theta$ to obtain $\mathcal{O}_{\theta}$, the wedges $W_{i, j}$ also rotate. Thus, the sets $\mathcal{W}^{i}$ change accordingly, giving rise to the sets $\mathcal{W}_{\theta}^{i}$. The rotated hull $\mathcal{O}_{\theta}$-hull of $P$ is then (recall Figure 18):

$$
\mathcal{O H}_{\theta}(P)=\mathbb{R}^{2} \backslash \bigcup_{i=1}^{2 k} \mathcal{W}_{\theta}^{i}
$$

Let $\partial\left(\mathcal{W}_{\theta}^{i}\right)$ denote the boundary of $\mathcal{W}_{\theta}^{i}$. As in Subsection 3.2.2 $\partial\left(\mathcal{W}_{\theta}^{i}\right)$ is an alternating polygonal chain, or staircase, with interior angle $\Theta_{i}=\pi-\alpha_{i}$ where, in counterclockwise direction around $\mathcal{O H}_{\theta}(P)$, right turns arise at apexes of $\mathcal{O}_{\theta}$-wedges in $\mathcal{W}_{\theta}^{i}$, called extremal, and left turns arise at points of $P$ which are the supporting points of those extremal wedges. Recall Figure 20 .

The following lemma follows directly from Lemma 5 .

Lemma 6. If two extremal $\mathcal{O}_{\theta}$-wedges have nonempty intersection, then they have to be opposite. When this happens, $\mathcal{O H}_{\theta}(P)$ gets disconnected.

As in Subsection 3.2.2, let $\Theta=\min \left\{\Theta_{i}: i=1, \ldots, 2 k\right\}$ for $\Theta_{i}=\pi-\alpha_{i}$, and again we will consider the two cases above.

The first case is where $\Theta \geq \frac{\pi}{2}$. We next show how to maintain $\mathcal{O H}_{\theta}(P)$ for $\theta \in[0,2 \pi)$. As in Subsection 2.2, we will denote by $\mathcal{S}_{\theta}(P)$ the set of overlapping regions in $\mathcal{O H}_{\theta}(P)$, and by $\mathcal{V}_{\theta}(P)$ the set of vertices of $\mathcal{O H}_{\theta}(P)$ in counterclockwise order while traversing $\partial\left(\mathcal{W}_{\theta}^{i}\right), i=1, \ldots, 2 k$.

Applying a rotation of angle $\theta$ to the set $\mathcal{O}$ changes the $\mathcal{O H}_{\theta}(P)$. In particular, the supporting vertices of the staircases $\partial\left(\mathcal{W}_{\theta}^{i}\right)$ might change. We now aim to update those staircases, in $O(\log n)$ time per insertion or deletion of a point. In order to do so, we need to maintain the (at most) $2 k$ staircases into (at most) $2 k$ different balanced trees, one for each staircase. Notice that some of the staircases may appear and/or disappear during the rotation. The total insertion or deletion operations can be done in $O(k n \log (k n))=O(k n \log n)$ time.

Using the circular table in Figure 21, we can rotate the (gray) stabbing wedges $W_{i+1, i+k}$, stopping at events arising when a defining ray of a stabbing wedge hits a vertex event in the innermost circle, i.e., entering or leaving an escaping interval (black). This provides the information about whether the stabbing wedges fit or not into the escaping intervals and this, as in Subsection 3.2.2, allows to handle the insertion or deletion of points in the set $\mathcal{V}_{\theta}(P)$ of vertices of $\mathcal{O H}_{\theta}(P)$ (i.e., the points on the staircases). Since the number of escaping intervals for a point is at most three and during the rotation these can arise in any of the $2 k$ wedges corresponding to rotated $W_{i+1, i+k}$, there are $O(k n)$ events. Thus, we get the following result. 
Theorem 8. Given a set $\mathcal{O}$ of $k$ lines such that $\Theta \geq \frac{\pi}{2}$, computing and maintaining the boundary of $\mathcal{O H}_{\theta}(P)$ during a complete rotation for $\theta \in[0,2 \pi)$ can be done in $O(k n \log n)$ time and $O(k n)$ space.

Furthermore, it is easy to see that while running our algorithm, we can keep track of the parameters in the following result.

Corollary 1. Given a set $\mathcal{O}$ of $k$ lines such that $\Theta \geq \frac{\pi}{2}$, computing an orientation $\theta$ such that the boundary of $\mathcal{O H}_{\theta}(P)$ has minimum number of steps, or minimum number of staircases, or it is connected, or it has the minimum number of connected components, can be done in $O(k n \log n)$ time and $O(k n)$ space.

Now, as in Section 3.2, we can also handle the second case where $\Theta<\frac{\pi}{2}$.

Theorem 9. Given a set $\mathcal{O}$ of $k$ lines such that $\Theta<\frac{\pi}{2}$, computing and maintaining the boundary of $\mathcal{O H}_{\theta}(P)$ during a complete rotation for $\theta \in[0,2 \pi)$ can be done in $O\left(k \frac{n}{\Theta} \log n\right)$ time and $O\left(k \frac{n}{\Theta}\right)$ space.

Corollary 2. Given a set $\mathcal{O}$ of $k$ lines such that $\Theta<\frac{\pi}{2}$, computing an orientation $\theta$ such that the boundary of $\mathcal{O H}_{\theta}(P)$ has minimum number of steps, or minimum number of staircases, or it is connected, or it has the minimum number of connected components, can be done in $O\left(k \frac{n}{\Theta} \log n\right)$ time and $O\left(k \frac{n}{\Theta}\right)$ space.

Another interesting consequence of Theorem 9 is that we can also generalize Theorem 1 to the case where $k=2$ and the two lines are not perpendicular.

Corollary 3. For a set $\mathcal{O}$ given by two non-perpendicular lines, computing and maintaining the boundary of $\mathcal{O H}_{\theta}(P)$ during a complete rotation for $\theta \in[0,2 \pi)$ can be done in $O\left(\frac{n}{\Theta} \log n\right)$ time and $O\left(\frac{n}{\Theta}\right)$ space, where $\Theta$ is the smallest aperture angle of the sectors defined by the two lines.

\subsection{Finding the value of $\theta$ for which $\mathcal{O H}_{\theta}(P)$ has minimum area}

The results in Subsections 2.2 and 2.3 can be adapted to the case of a set $\mathcal{O}$ of $k$ lines through the origin such that all the sectors they define have aperture angle at most $\frac{\pi}{2}$, i.e., with $\Theta \geq \frac{\pi}{2}$. Again, for a fixed value of $\theta$, we can compute the area of $\mathcal{O H}_{\theta}(P)$ using the fact that

$$
\operatorname{area}\left(\mathcal{O H}_{\theta}(P)\right)=\operatorname{area}(\mathcal{P}(\theta))-\operatorname{area}\left(\mathcal{P}(\theta) \backslash \mathcal{O H}_{\theta}(P)\right),
$$

where $\mathcal{P}(\theta)$ denotes the polygon having the points in $\mathcal{V}_{\theta}(P)$ as vertices and an edge connecting two vertices if they are consecutive elements in $\mathcal{V}_{\theta}(P)$. Again, we will compute the area of $\mathcal{P}(\theta) \backslash \mathcal{O H}_{\theta}(P)$ by decomposing it into two types of regions: (i) The triangles defined by every pair of consecutive elements in $\mathcal{V}_{\theta}(P)$, and (ii) those rhomboid overlaps between two triangles which make $\mathcal{O H}_{\theta}(P)$ be disconnected. Recall Figures 3 and 20.

By Theorem 8, the triangles in (i) above can be maintained in $O(k n \log n)$ time and $O(k n)$ space. While $\theta$ increases from 0 to $2 \pi$, the set $\mathcal{V}_{\theta}(P)$ of points on $\partial\left(\mathcal{O H}_{\theta}(P)\right)$ changes at the values of $\theta$ where a point of $P$ becomes (resp. is no longer) a vertex of $\mathcal{O H}_{\theta}(P)$. These angles are again called insertion (resp. deletion) events.

Next, we will deal with the rhomboids in (ii), showing how to maintain the set $\mathcal{S}_{\theta}(P)$ of rhomboid overlaps which, analogously to $\mathcal{V}_{\theta}(P)$, changes at overlap (resp. release) events. We will use the same techniques as in Subsection 2.2, but repeating the process at most $k$ times, i.e., computing separately all the possible overlapping rhomboids for each pair of opposite staircases in $\mathcal{O H}_{\theta}(P)$ while $\theta$ increases in $[0,2 \pi)$. 


\subsubsection{The sequence of overlap and release events}

Let us denote an overlap as $S_{\theta}(i, j)$, and let $\mathcal{S}_{\theta}(P)$ be the set of all overlaps $S_{\theta}(i, j)$ of $\mathcal{O H}_{\theta}(P)$, for angle $\theta$. By Theorem 8 , for a fixed value of $\theta, \mathcal{O H}_{\theta}(P)$ can be computed in $O(k n \log n)$ time and $O(k n)$ space, and then $\mathcal{S}_{\theta}(P)$ can be computed from $\mathcal{V}_{\theta}(P)$ in $O(n)$ time.

To maintain the set $\mathcal{S}_{\theta}(P)$ while $\theta$ increases from 0 to $2 \pi$, we will compute a sequence of events for each pair of opposite staircases, computing their overlap and release events. The events of each pair are computed independently, and then the $k$ sequences are merged in $O(k n)$ time to form the definitive sequence of all overlap and release events.

By Lemma 6, only opposite staircases $\partial\left(\mathcal{W}_{\theta}^{i}\right)$ and $\partial\left(\mathcal{W}_{\theta}^{i+k}\right)$ can intersect, i.e., for any $\theta$, only one pair $\{i, i+k\}$ of opposite staircases can intersect, making $\mathcal{O H}_{\theta}(P)$ disconnected. The corresponding intersection can be composed of several overlapping regions, which we will show next how to maintain as $\theta$ increases.

We will adapt the definitions and observations in Subsection 2.2.2, highlighting the differences. First, the chain of arcs is defined in the same way, with the only difference that the locus of the points being apexes of $P$-free extremal $\theta$-wedges might be "flatter arcs", with curvature smaller than or equal to that of circle arcs, since the aperture angles of the wedges are now at least $\frac{\pi}{2}$ instead of exactly $\frac{\pi}{2}$. Naturally, the possibility of the arcs being flatter does not affect to the monotonicity of a sub-chain $A_{e_{i}}$ with respect to an edge $e_{i}$ of $\partial(\mathcal{C H}(P))$. The property of overlapping regions corresponding to intersecting links is also maintained. The reader might recall Figure 5, considering the possibility that flatter arcs appear.

Next, we adapt Subsection 2.2 .3 to see that the number of intersections between links is now in $O(k n)$. For that, it is enough to check that Lemmas 2, 3, and 4 are valid for each of the $k$ pairs of opposite staircases in $\mathcal{O H}_{\theta}(P)$ and, using them, Theorem 2 is still true for each of the $O(k)$ pairs of opposite staircases in $\mathcal{O H}_{\theta}(P)$. It is important to notice that the assumption of $\Theta \geq \frac{\pi}{2}$ in this Subsection 3.4 is needed for these two last paragraphs to work.

Then, we can adapt Subsection 2.2.4 to compute the sequence of overlap and release events. In order to do so, we proceed with an algorithm analogous to the one outlined there, but computing in $O(n \log n)$ time and $O(n)$ space the sequence of overlap and release events for each pair of opposite staircases. After doing so, we merge the $k$ sequences of overlap and release events obtained into a single sequence of overlap and release events, obtaining the sorted events during a complete rotation of $\theta$ from 0 to $2 \pi$. In this way, we get the following result, which generalizes Theorem 3 .

Theorem 10. The sequence of overlap and release events for $\mathcal{O H}_{\theta}(P)$, while $\theta$ increases from 0 to $2 \pi$, can be computed in $O(k n \log n)$ time and $O(k n)$ space.

In order to sweep the sequence of overlap and release events, we store again the events sequence as points on a circle $[0,2 \pi)$, over which we represent the wedges $W_{i+1, i+k}$ in a similar way as we did in the innermost circle in Figure 21, right (where we stored the vertex events instead). Proceeding as in Subsection 2.2.5, but considering that now we have at most $O(k n)$ total overlap and release events, we get the following result, which generalizes Theorem 4 .

Theorem 11. Using the sequence of overlap and release events for $\mathcal{O H}_{\theta}(P)$, the set $\mathcal{S}_{\theta}(P)$ can be maintained while $\theta$ increases in $[0,2 \pi)$ using $O(k n)$ time and $O(k n)$ space.

\subsubsection{Computing minimum area}

The final step is to compute the value of $\theta$ that minimizes (or maximizes) the area of $\mathcal{O H}_{\theta}(P)$. We show next how to compute this angle in $O(k n \log n)$ time and $O(k n)$ space. 
Let $\alpha, \beta$ be two events (of any type) such that $(\alpha, \beta)$ is an angular interval in $[0,2 \pi)$ containing no events. Extending Equation 9 and mimicking Equation 3, we express the area of $\mathcal{O H}_{\theta}(P)$ for any $\theta \in(\alpha, \beta)$ as follows:

$$
\operatorname{area}\left(\mathcal{O H}_{\theta}(P)\right)=\operatorname{area}(\mathcal{P}(\theta))-\sum_{i} \operatorname{area}\left(\triangle_{i}(\theta)\right)+\sum_{j} \operatorname{area}\left(\diamond_{j}(\theta)\right) .
$$

As before, $\mathcal{P}(\theta)$ denotes the polygon having the points in $\mathcal{V}_{\theta}(P)$ as vertices, and an edge connecting two vertices if they are consecutive elements in $\mathcal{V}_{\theta}(P)$. The term $\triangle_{i}(\theta)$ denotes a triangle defined by two consecutive vertices $p, q \in \mathcal{V}_{\theta}(P)$. The boundary of this triangle is formed by the line segment $\overline{p q}$ and one of the current $\mathcal{O}_{\theta}$-wedges in $\mathcal{W}_{\theta}^{i}$ supported by $p$ and $q$. As the aperture angle of an extremal wedge is at least $\frac{\pi}{2}$, the triangle is now either rectangular (Figure 3) or obtuse (Figure 22). Finally, the term $\diamond_{j}(\theta)$ denotes the $j$-th overlapping region in $\mathcal{S}_{\theta}(P)$, which is now either a rectangle (Figure 3) or a rhomboid (Figure 23).

We now show that, for any particular value of $\theta$, we can evaluate Equation $(10)$ in $O(k n \log n)$ time and, as $\theta$ increases from 0 to $2 \pi$, a constant number of terms need to be updated at each event, regardless of its type.

The polygon. At any fixed value of $\theta$ the area of $\mathcal{P}(\theta)$ can be computed from $\mathcal{V}_{\theta}(P)$ in $O(n)$ time. The term area $(\mathcal{P}(\theta))$ changes only at vertex events. These events can be processed in constant time, since at an insertion (resp. deletion) event, the area of a single triangle needs to be subtracted (resp. added) to the previous value of area $(\mathcal{P}(\theta))$, as the same as in Figure 14 .

The triangles. Let $p$ and $q$ be two consecutive vertices in $\mathcal{V}_{\theta}(P)$ such that $p$ precedes $q$. Suppose that, for any $\theta \in(\alpha, \beta)$, the points $p$ and $q$ define the triangle $\triangle_{i}(\theta)$. This triangle is bounded by $\overline{p q}$ and an extremal wedge supported by $p$ and $q$. Let $\omega \geq \frac{\pi}{2}$ denote the aperture angle of the extremal wedge, and $\omega_{p}$ and $\omega_{q}$ denote respectively, the internal angles of $\triangle_{i}(\alpha)$ at $p$ and $q$. See Figure 22 .

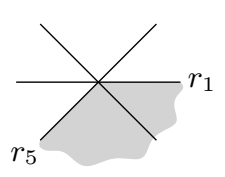

(a)

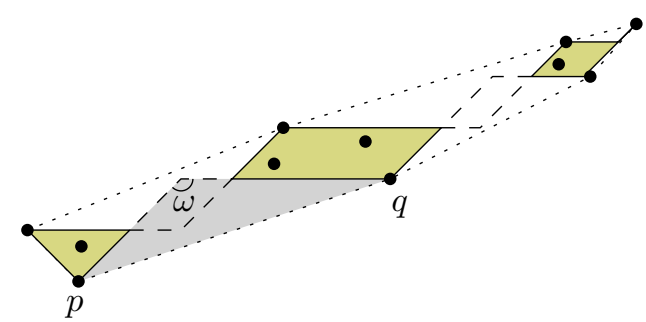

(b)

Figure 22: Computing the area of a triangular region. (a) The wedge of size $\omega$ that bounds the triangle. (b) The triangle defined by $p$ and $q$.

The area of $\triangle_{i}(\theta)$ can be expressed as

$$
\operatorname{area}\left(\triangle_{i}(\theta)\right)=\frac{|\overline{p q}|^{2}}{2 \sin (\omega)} \cdot \sin \left(\omega_{p}+(\theta-\alpha)\right) \cdot \sin \left(\omega_{q}-(\theta-\alpha)\right),
$$

and expanding Equation (11) we obtain

$$
\begin{aligned}
\operatorname{area}\left(\triangle_{i}(\theta)\right) & =A \cdot \cos ^{2}(\theta-\alpha)+B \cdot \cos (\theta-\alpha) \cdot \sin (\theta-\alpha)+C \cdot \sin ^{2}(\theta-\alpha) \\
& =D+E \cdot \cos (2(\theta-\alpha))+F \cdot \sin (2(\theta-\alpha)),
\end{aligned}
$$


where $A \ldots F$ are constant values in terms of $\omega, \omega_{p}, \omega_{q}$, and the coordinates of $p$ and $q$. Therefore, the term $\sum_{i}$ area $\left(\triangle_{i}(\theta)\right)$ is linear in $\cos (2(\theta-\alpha))$ and $\sin (2(\theta-\alpha))$. Since each point of $P$ can appear in $O(k)$ staircases we have $O(k n)$ triangles, which can be processed in $O(k n)$ time as we described in Subsection 2.2.

The overlapping regions. Let $p, q$ be two consecutive vertices in $\mathcal{V}_{\theta}(P)$ such that $p$ precedes $q$, and let $r, s$ be two consecutive vertices in $\mathcal{V}_{\theta}(P)$ such that $r$ precedes $s$. Suppose that, for any $\theta \in(\alpha, \beta)$, the points $p, q, r, s$ define the overlapping region $\diamond_{j}(\theta)$. Without loss of generality, we assume that $p$ and $q$ support an extremal wedge in $\mathcal{W}^{i}$, and that $r$ and $s$ support an extremal wedge in $\mathcal{W}^{i+k}$. We denote with $\omega \geq \frac{\pi}{2}$ the aperture angle of both wedges. See Figure 23 (a).

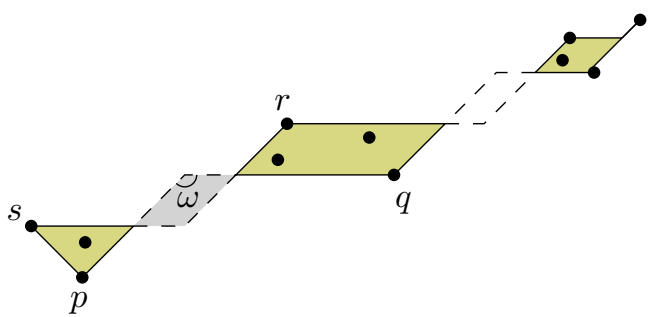

(a)

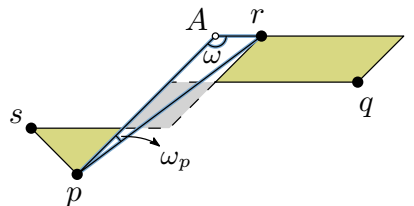

(b)

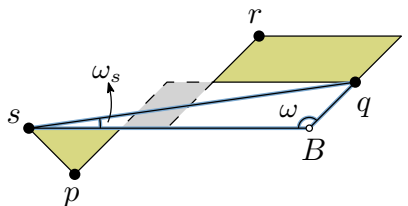

(c)

Figure 23: Computing the area of an overlapping region. (a) The overlapping region defined by $p, q, r$, and $s$. (b) The triangle $\triangle p r A$. (c) The triangle $\triangle q s B$.

Let us consider the overlapping region when $\theta=\alpha$. Based on the triangles $\triangle \operatorname{pr} A$ (Figure 23(b) and $\triangle q s B$ (Figure 23.(c)), the area of $\diamond_{j}(\theta)$ can be expressed as

$$
\diamond_{j}(\theta)=\frac{|\overline{p r}| \cdot|\overline{q s}|}{\sin ^{2}(w)} \cdot \sin \left(\omega_{p}+(\theta-\alpha)\right) \cdot \sin \left(\omega_{s}-(\theta-\alpha)\right),
$$

and expanding Equation 13 we obtain

$$
\begin{aligned}
\operatorname{area}\left(\triangle_{i}(\theta)\right) & =A^{\prime} \cdot \cos ^{2}(\theta-\alpha)+B^{\prime} \cdot \cos (\theta-\alpha) \cdot \sin (\theta-\alpha)+C^{\prime} \cdot \sin ^{2}(\theta-\alpha) \\
& =D^{\prime}+E^{\prime} \cdot \cos (2(\theta-\alpha))+F^{\prime} \cdot \sin (2(\theta-\alpha)),
\end{aligned}
$$

where $A^{\prime} \ldots F^{\prime}$ are constant values in terms of $\omega, \omega_{p}, \omega_{s}$, and the coordinates of $p, q, r$, and $s$. The term $\sum_{i}$ area $\left(\diamond_{i}(\varphi)\right)$ is therefore linear in $\cos (2(\theta-\alpha))$ and $\sin (2(\theta-\alpha))$. We thus can have at most $O(k n)$ overlapping regions in the sequence of overlap and release events, so they can be processed in $O(k n)$ time as in Subsection 2.3. Notice that the area of a rhomboid can be computed in constant time analogously to the area of a rectangle, so the formulas there are analogous. Using the algorithm in Subsection 2.3.4 with the mentioned changes in the number of events and complexities, we get our final result.

Theorem 12. Given a set $\mathcal{O}$ of $k$ lines such that $\Theta \geq \frac{\pi}{2}$, computing $\mathcal{O H}_{\theta}(P)$ with minimum (or maximum) area over all $\theta \in[0,2 \pi)$ can be done in $O(k n \log n)$ time and $O(k n)$ space.

\section{References}

[1] J. Abello, V. Estivill-Castro, T. Shermer, and J. Urrutia. Illumination of orthogonal polygons with orthogonal floodlights. International Journal of Computational Geometry E Applications, 8(1), (1998), 25-38. 
[2] C. Alegría-Galicia, T. Garduño, A. Rosas-Navarrete, C. Seara, and J. Urrutia. Rectilinear convex hull with minimum area. Special LNCS Festschrift volume in honor of Ferran Hurtado's 60th Birthday. Lecture Notes in Computer Science, Vol. 7579, (2012), 226-235.

[3] C. Alegría-Galicia, C. Seara, and J. Urrutia. Computing containment relations between rectilinear convex hulls. Mexican Conference on Discrete Mathematics and Computational Geometry, 60th birthday of Jorge Urrutia, (2013).

[4] C. Alegría-Galicia, D. Orden, C. Seara, and J. Urrutia. On the $\mathcal{O}$-hull of planar point sets. 30th European Workshop on Computational Geometry (EuroCG), (2014).

[5] C. Alegría-Galicia, D. Orden, C. Seara, and J. Urrutia. Optimizing an oriented convex hull with two directions. 31th European Workshop on Computational Geometry (EuroCG), (2015).

[6] C. Alegría-Galicia, D. Orden, C. Seara, and J. Urrutia. On the $\mathcal{O}_{\beta}$-hull of a planar point set. Computational Geometry: Theory and Applications, 68, (2018), 277-291.

[7] D. Avis, B. Beresford-Smith, L. Devroye, H. Elgindy, E. Guévremont, F. Hurtado, and B. Zhu. Unoriented $\Theta$-maxima in the plane: Complexity and algorithms. SIAM J. Comput., 28(1), (1999), 278-296.

[8] S. W. Bae, Ch. Lee, H.-K. Ahn, S. Choi, Sunghee, and K.-Y. Chwa. Computing minimumarea rectilinear convex hull and L-shape. Computational Geometry: Theory and Applications, 42(9), (2009), 903-912.

[9] J. L. Bentley and T. A. Ottmann. Algorithms for reporting and counting geometric intersections. IEEE Transactions on Computers, 4059, (2006), 643-647.

[10] T. Biedl and B. Genç. Reconstructing orthogonal polyhedra from putative vertex sets. Computational Geometry: Theory and Applications, 44(8), (2011), 409-417.

[11] A. Biswas, P. Bhowmick, M. Sarkar, and B. B. Bhattacharya. Finding the orthogonal hull of a digital object: A combinatorial approach. Proceedings of the 12th International Conference on Combinatorial Image Analysis, IWCIA'08, (2008), 124-135.

[12] J. M. Díaz-Báñez, M. A. López, M. Mora, C. Seara, and I. Ventura. Fitting a two-joint orthogonal chain to a point set. Computational Geometry: Theory and Applications, 44(3), (2011), 135-147.

[13] M. Dutt, A. Biswas, P. Bhowmick, and B. B. Bhattacharya. On finding an orthogonal convex skull of a digital object. International Journal of Imaging Systems and Technology, 21(1), (2011), 14-27.

[14] E. Fink and D. Wood. Restricted-orientation Convexity. Monographs in Theoretical Computer Science (An EATCS Series), Springer-Verlag (2004).

[15] V. Franěk and J. Matoušek. Computing D-convex hulls in the plane. Computational Geometry: Theory and Applications, 42(1), (2009), 81-89.

[16] R. H. Güting. Conquering contours: efficient algorithms for computational geometry. PhD thesis, Fachbereich Informatik, Universität Dortmund, (1983).

[17] H.-T. Kung, F. Luccio, and F. P. Preparata. On finding the maxima of a set of vectors. Journal of the ACM, 22(4), (1975), 469-476. 
[18] T. van Lankveld, M. van Kreveld, and R. Veltkamp. Identifying rectangles in laser range data for urban scene reconstruction. Computers \& Graphics, 35(3), (2011), 719-725.

[19] J. Matoušek and P. Plecháč. On functional separately convex hulls. Discrete $\&$ Computational Geometry, 19(1), (1998), 105-130.

[20] D. Y. Montuno and A. Fournier. Finding XY convex hull of a set of XY polygons. Computer Systems Research Group, University of Toronto, (1982).

[21] T. N. Nicholl, D.-T. Lee, Y.-Z. Liao, and Ch.-K. Wong. On the XY convex hull of a set of XY polygons. BIT Numerical Mathematics, 23(4), (1983), 456-471.

[22] T. Ottmann, E. Soisalon-Soininen, and D. Wood. On the definition and computation of rectilinear convex hulls. Information Sciences, 33(3), (1984), 157-171.

[23] F. P. Preparata and M. I. Shamos. Computational Geometry: An introduction, SpringerVerlag, (1985).

[24] D. S. Raicu, J. D. Furst, D. Channin, D.-Hui Xu, A. Kurani, and S. Aioaneid. A texture dictionary for human organs tissues' classification. Proceedings of the 8th World Multiconference on Systemics, Cybernetics and Informatics (SCI 2004), (2004).

[25] G. J. E. Rawlins and D. Wood. Ortho-convexity and its generalizations. Computational Morphology: A Computational Geometric Approach to the Analysis of Form, NorthHolland, (1988).

[26] F. Sheikhi, A. Mohades, M. de Berg, and M. Davoodi. Separating bichromatic point sets by L-shapes. Computational Geometry: Theory and Applications, 48(9), (2015), 673-687.

[27] W. Son, S.-w Hwang, and H.-K. Ahn. MSSQ: Manhattan spatial skyline queries. Information Systems, 40, (2014), 67-83.

[28] E. Uchoa, M. Poggi de Aragão, and C. Ribeiro. Preprocessing steiner problems from VLSI layout. Networks, 40(1), (1999), 38-50.

[29] X. Zhang, Z. Tang, J. Yu, M. Guo, and L. Jiang. Convex hull properties and algorithms. Applied Mathematics and Computation, 216(11), (2010), 3209-3218. 